\title{
An experimental study of localized bulging in inflated cylindrical tubes guided by newly emerged analytical results
}

\author{
Shibin Wang ${ }^{\mathrm{a}}$, Zhiming Guo ${ }^{\mathrm{a}}$, Lei Zhou ${ }^{\mathrm{a}}$, Linan $\mathrm{Li}^{\mathrm{a}}$, Yibin $\mathrm{Fu}^{\mathrm{a}, \mathrm{b}, *}$ \\ ${ }^{a}$ Department of Mechanics, Tianjin University, Tianjin 300072, China \\ ${ }^{b}$ School of Computing and Mathematics, Keele University, Staffordshire ST5 5BG, UK
}

\begin{abstract}
An experimental study is conducted on localized bulging of inflated latex rubber tubes of a range of wall thicknesses and tube lengths, guided by newly emerged analytical results. In the case when the tube has one free closed end that may or may not be subjected to a dead weight, the initiation pressure for localized bulging is determined by a bifurcation condition, and the propagation pressure is determined by Maxwell's equal-area rule. It is shown that after bulge initiation the pressure will decrease monotonically towards, but will never reach, the propagation pressure, and it is when the pressure is sufficiently close to this propagation pressure that rapid propagation of the bulge in the axial direction takes place. It is found that the experimentally observed initiation pressure is around $15 \%$ below the theoretical prediction, which is consistent with the fact that bulging initiation is a subcritical phenomenon and is therefore sensitive to imperfections. The experimentally observed propagation pressure is always very close to the theoretical prediction, which confirms the insensitivity of this pressure to imperfections and demonstrates the predictive power of the material model fitted from our own experiments on equibiaxial stretching. In the other case when the tube is first stretched and then fixed at both ends, bulge initiation takes place in the same manner as in the previous case, but the propagation pressure is no longer determined by Maxwell's equal-area rule. After bulge initiation the pressure will first decrease to a minimum and then rises slowly, and it is on the latter ascending path that the bulge starts to propagate rapidly in the axial direction. A semi-analytical method is proposed for the determination of the minimum pressure. Numerical simulations with the use of the software Abaqus are also conducted to verify the theoretical predictions.
\end{abstract}

Keywords: Localized bulging, rubber tubes, aneurysm, bifurcation, nonlinear elasticity.

\section{Introduction}

Localized bulging in an inflated cylindrical tube is an important prototypical problem that exhibits the three distinct phases of initiation, growth, and propagation shared by a

${ }^{*}$ Corresponding author at: School of Computing and Mathematics, Keele University, Staffordshire ST5 $5 \mathrm{BG}, \mathrm{UK}$

Email address: y.fu@keele.ac.uk (Yibin Fu) 
variety of other localization problems (Kyriakides and Chang 1990). The earliest study dates back to Mallock (1891) who used the existence of a pressure maximum in uniform inflation as an explanation for this phenomenon. Subsequently, this topic has continuously been studied both theoretically, numerically, and experimentally; see Yin (1977), Chater and Hutchinson (1984), Kyriakides and Chang (1990, 1991), Shi and Moita (1996), Gent (2005), Pamplona et al (2006), Goncalves et al (2008), and the references therein. The topic is relevant to a variety of applications, and is experiencing a revival due to increased attention paid to human health and use of soft devices in hi-tech applications. Recent studies include continuum-mechanical modelling of aneurysm initiation/suppression in human arteries and energy harvesting devices (Ren 2007; Fu et al 2012; Bucchi and Hearn 2013a,b; Alhayani et al. 2013, 2014; Rodrguez-Martnez it et al 2015; Varatharajan and DasGupta 2017), and localized bulging under the additional effects of swelling (Demirkoparan and Merodio 2017), viscoelasticity/chemorheology (Wineman 2015, 2017), plasticity (Takla 2018), and electric actuation (Lu et al 2015; An et al 2015; Wang et al 2017). The topic is closely related to the so-called limiting point instability that has also been much studied in the literature; see, for instance, Alexander (1971), Kanner and Horgan (2007), Mao et al (2014), Horny et al (2015), Wang et al (2018), and the references therein. It is also an ideal testing ground for strain-gradient theories (Lestringant and Audoly 2018).

On the theoretical side, since localized bulging was first recognized as a bifurcation phenomenon (Fu et al 2008), much progress has been made in understanding the roles played by imperfections (Fu and Xie 2012), constitutive laws (Pearce 2012), wall thickness (Fu et al 2016), fibre-reinforcement (Wang and Fu 2018), an internal mean flow (Fu and Ilichev 2015), and surface texture (Guo et al 2016). In particular, we highlight the following main results: (i) localized bulging is a subcritical bifurcation phenomenon and so is very sensitive to imperfections; (ii) although in the case of fixed axial force the initiation pressure corresponds to the maximum pressure in uniform inflation, localized bulging in the case of fixed axial length is much less intuitive: it is possible even if pressure in uniform inflation is monotonic, and the propagation pressure is no longer determined by Maxwell's equal area rule; (iii) if the tube is isotropic, then theoretical predictions for the initiation and propagation pressures based on the membrane theory are valid even for moderately thick tubes, but when fibre-reinforcement is introduced, the membrane theory must be used with caution.

There exist two major sets of experimental studies, Kyriakides and Chang (1990, 1991), Pamplona et al (2006) and Goncalves et al (2008), that are concerned with fixed axial force and fixed axial length, respectively; these studies have already provided a lot of insight into the bulging phenomenon. The case of fixed axial force refers to the situation where one end of the tube is closed and is free from any displacement restrictions although a dead weight may be attached to exert an extra stretching force; see Figure 11. The other case refers to the situation where the tube is first stretched and then both ends are fixed when inflation is carried out; see Figure 18. Our current work is intended to complement what has been 
achieved previously, and is motivated by the fact that the above-mentioned experimental studies were carried out without the benefit of the theoretical results that have only emerged more recently, and as a result comparison with theory in these studies is inevitably limited in its scope. For instance, when Kyriakides and Chang conducted their experiments (Kyriakides and Chang 1990, 1991), it was not yet known what the theoretical initiation pressure should be and how sensitive it is to imperfections, and so they compared their initiation pressure with the critical pressure associated with the first sinusoidal bifurcation mode. When Pamplona et al (2006) and Goncalves et al (2008) conducted their experiments more recently for the case of fixed axial length, they were only able to compare their experimental studies with a numerical simulation since it was not yet known how the initiation and minimum pressures could be determined. In the present study, we conduct a new set of experiments, guided by the analytical results that are now available. Comparisons between experiments and theoretical predictions are made in order to elucidate the imperfection sensitivity of the initiation pressure, the robustness of the propagation pressure, and how these results depend on end conditions (whether it is the axial force or axial length that is fixed). Our objective is to update the existing experimental studies and present state of the art on this topic.

The rest of this paper is organized as follows. After formulating the problem and summarizing the necessary analytical results, we describe in Section 2 a semi-analytical method for determining the minimum pressure in the case of fixed axial length. In Section 3 we explain our experimental set-up and material characterization. This is then followed in Section 4 by a detailed comparison between the theoretical predictions and experimental results, aided also by numerical simulations. The paper is concluded with a summary and some further discussions.

\section{Problem formulation and summary of analytical results}

Consider a hyperelastic, isotropic, and incompressible cylindrical tube that initially has inner radius $A$, outer radius $B$, and length $2 L$. When it is uniformly stretched in the axial direction and inflated by an internal pressure $P$, the inner and outer radii become $a$ and $b$, respectively. The three principal stretches associated with the uniform inflation are given by

$$
\lambda_{1} \equiv \lambda=\frac{r}{R}, \quad \lambda_{2}=\lambda_{z}, \quad \lambda_{3}=1 /\left(\lambda_{1} \lambda_{2}\right),
$$

where $R$ and $r$ are the radial coordinates in the initial and inflated configurations, respectively, and $\lambda_{z}$ is the constant stretch in the z-direction. The dependence of $r$ on $R$ can be determined by the condition of incompressibility.

We assume that the constitutive behavior of the tube is described by a strain-energy function $W\left(\lambda_{1}, \lambda_{2}, \lambda_{3}\right)$, and define a reduced strain-energy function $w$ by

$$
w\left(\lambda, \lambda_{z}\right)=W\left(\lambda, \lambda_{z}, \lambda^{-1} \lambda_{z}^{-1}\right)
$$


Then the internal pressure $P$ and the net axial force $F$ at any cross section are given by

$$
\begin{gathered}
P=\int_{\lambda_{b}}^{\lambda_{a}} \frac{w_{1}}{\lambda^{2} \lambda_{z}-1} d \lambda, \\
F \equiv 2 \pi \int_{a}^{b} \sigma_{22} r d r-\pi a^{2} P=\pi A^{2}\left(\lambda_{a}^{2} \lambda_{z}-1\right) \int_{\lambda_{b}}^{\lambda_{a}} \frac{2 \lambda_{z} w_{2}-\lambda w_{1}}{\left(\lambda^{2} \lambda_{z}-1\right)^{2}} \lambda d \lambda,
\end{gathered}
$$

where $w_{1}=\partial w / \partial \lambda, w_{2}=\partial w / \partial \lambda_{z}, \sigma_{22}$ is the principal Cauchy stress in the axial direction, and the two limits $\lambda_{a}$ and $\lambda_{b}$ are defined by

$$
\lambda_{a}=\frac{a}{A}, \quad \lambda_{b}=\frac{b}{B} .
$$

See, e.g., Haughton and Ogden (1979). It follows from incompressibility that $\lambda_{a}$ and $\lambda_{b}$ are related to each other by

$$
\lambda_{a}^{2} \lambda_{z}-1=\frac{B^{2}}{A^{2}}\left(\lambda_{b}^{2} \lambda_{z}-1\right)
$$

With $\lambda_{b}$ eliminated with the use of this equation, $P$ and $F$ can both be viewed as functions of $\lambda_{a}$ and $\lambda_{z}$. It was shown in Fu et al (2016) that the bifurcation condition for localized bulging takes the simple form

$$
J(P, F) \equiv \frac{\partial P}{\partial \lambda_{a}} \frac{\partial F}{\partial \lambda_{z}}-\frac{\partial P}{\partial \lambda_{z}} \frac{\partial F}{\partial \lambda_{a}}=0,
$$

which states that the Jacobian of $P$ and $F$ vanishes. Mathematically, this is the condition under which the expressions for $P$ and $F$ can no longer be inverted to express $\lambda_{a}$ and $\lambda_{z}$ uniquely in terms of $P$ and $F$. Other forms of this bifurcation condition can be found in $\mathrm{Fu}$ et al (2018).

We observe that the bifurcation condition (2.5) is independent of the conditions imposed at the ends of the tube. In the experimental studies of Kyriakides and Chang (1990, 1991), Pamplona et al (2006) and Goncalves et al (2008), the two typical cases of fixed $F$ and fixed axial length are considered, respectively. If, for instance, one of the two ends is closed, and is pulled by a dead weight with unrestricted movement, then $F$ is fixed and is equal to the dead weight. In this case $\lambda_{z}$ can be expressed in terms of $\lambda_{a}$ with the use of (2.3), and it can then be shown (Fu and Ilichev 2015) that the bifurcation condition reduces to $d P / d v=0$ where $v$ is the enclosed volume in the deformed configuration. The propagation pressure is determined by Maxwell's equal area rule (Chater and Hutchinson 1984). Thus, in this case a necessary condition for localized bulging is that the inflation pressure as a function of $v$ has a limiting/turning point, and the bulge will start to propagate rapidly in the axial direction after having almost reached a maximum radius (or equivalently, when the pressure is sufficiently close to its Maxwell value). We remark that the maximum radius can never be reached exactly since a kink state (or a heteroclinic orbit) can only exist in an infinite domain. As the maximum radius is approached, a small further reduction in pressure will induce a large extension of the bulged section.

In the other case of fixed axial length, the tube is first stretched from its original length $2 L$ to $\lambda_{z 0}(2 L)$, say, and the latter length is then fixed during subsequent inflation. With 
end effects neglected, the initial inflation may be considered to be uniform with fixed axial stretch $\lambda_{z 0}$. However, as soon as a bulge has initiated, the $\lambda_{z}$ near the two ends will decrease (i.e. unloading will take place) even though it may still be taken to be approximately independent of $Z$ outside a boundary layer. Bulging can take place even if $P$ in uniform inflation is monotonic (as for arteries), and the propagation pressure is no longer determined by the equal area rule. This will be discussed in more detail later.

Defining the wall thickness $H$, averaged radius $R_{m}$, and scaled wall thickness $\varepsilon$ through

$$
H=B-A, \quad R_{m}=(A+B) / 2, \quad \varepsilon=H / R_{m},
$$

we may expand $(2.2),(2.3)$, and $(2.5)$ in terms of $\varepsilon$ to obtain

$$
P=\varepsilon \frac{w_{1}}{\lambda_{m} \lambda_{z}}+O\left(\varepsilon^{3}\right), \quad \frac{F}{2 \pi R_{m} H}=w_{2}-\frac{\lambda_{m} w_{1}}{2 \lambda_{z}}+O\left(\varepsilon^{2}\right)
$$

and

$$
\Omega^{(0)}+O\left(\varepsilon^{2}\right)=0, \quad \Omega^{(0)} \equiv \lambda_{m}\left(w_{1}-\lambda_{z} w_{12}\right)^{2}+\lambda_{z}^{2} w_{22}\left(w_{1}-\lambda_{m} w_{11}\right),
$$

where all the partial derivatives of $w$ are evaluated at the principal stretches in the midsurface where the stretch in the azimuthal direction is denoted by $\lambda_{m}$ (the axial stretch is still $\lambda_{z}$ ). The leading order terms in the above expansions correspond to the membrane theory. It was shown in $\mathrm{Fu}$ et al (2016) with the use of these expansions that the membrane theory gives good predictions for the initiation pressure for localized bulging, with a relative error less than $5 \%$, for values of $\varepsilon$ up to 0.67 . This will be confirmed by our experimental and numerical simulation results to be presented later.

In view of the expressions in (2.6), we shall in the rest of this paper scale all lengths by $R_{m}$, the pressure by $\mu \varepsilon$, and the axial force $F$ by $2 \pi R_{m} H \mu$, where $\mu$ is the ground state shear modulus. Thus, for instance, $r(0)$ will denote $r(0) / R_{m}$, and $\varepsilon=H$ from now on. The scaled $P$ and $F$ are then both independent of $H$ under the membrane assumption.

In the rest of this section, we shall use the membrane theory to explain the differences in the inflation behaviour between the two loading scenarios. The advantage of using the membrane theory is that the entire bulging process can be described analytically or semianalytically. In particular, we shall show how the minimum pressure in the case of fixed axial length can be computed semi-analytically. In this case the propagation pressure can only be estimated; it cannot be determined exactly.

To describe both the uniformly inflated and bulging solutions under the membrane assumption, we now consider a generally axisymmetric deformation described by

$$
r=r(Z), \quad \theta=\Theta, \quad z=z(Z), \quad-L \leq Z \leq L,
$$

where $r$ is now the deformed radius of the mid-surface scaled by $R_{m}$, and $Z$ and $z$ are the scaled axial coordinates in the initial and inflated configurations, respectively. The principal directions of stretch coincide with the lines of latitude, the meridian and the normal to the deformed (middle) surface. Thus, the principal stretches are now given by

$$
\lambda_{1}=r, \quad \lambda_{2}=\sqrt{r^{\prime 2}+z^{\prime 2}}, \quad \lambda_{3}=1 /\left(\lambda_{1} \lambda_{2}\right),
$$


where the indices $(1,2,3)$ are used for the latitudinal, meridional and normal directions respectively, and the primes indicate differentiation with respect to $Z$.

It can be shown that the equilibrium equations may be written in the form

$$
\begin{aligned}
\lambda_{1}^{\prime} & =\lambda_{2} \sin \phi \\
\lambda_{2}^{\prime} & =\frac{w_{1}-\lambda_{2} w_{12}}{w_{22}} \sin \phi, \\
\phi^{\prime} & =\frac{w_{1}}{w_{2}} \cos \phi-\frac{P \lambda_{1} \lambda_{2}}{w_{2}},
\end{aligned}
$$

where $\phi$ is the angle between the meridian and the Z-axis, and $w$ now denotes $H w / \mu$ (that is the strain energy per unit surface area in the undeformed configuration, scaled by the ground state shear modulus $\mu$ ). We assume that the crown of any bulging solution corresponds to $Z=0$. It can be deduced from $(2.10)_{3}$ that

$$
P=\frac{w_{1}}{\lambda_{1} \lambda_{2}}, \quad \text { wherever } \phi=0 \text { and } \phi^{\prime}=0 .
$$

In particular, this expression is valid at the crown of the bulge, or where the solution is uniform.

It is well-known (Pipkin 1968) that the above equilibrium equations have two integrals, given by

$$
w-\lambda_{2} w_{2}=K_{1}, \quad \frac{w_{2} z^{\prime}}{\lambda_{2}}-\frac{1}{2} P \lambda_{1}^{2}=K_{2},
$$

where $K_{1}$ and $K_{2}$ are integration constants. We are only concerned with localized bulging solutions, and will assume that the tube is sufficiently long so that the two principal stretches near the two ends may be assumed to be uniform, which we denote by $\lambda_{1 \infty}$ and $\lambda_{2 \infty}$. This assumption is justified by the fact that a bulging solution is effectively a static solitary wave with amplitude decaying exponentially away from its crown. The constants $K_{1}$ and $K_{2}$ can then be determined by evaluating (2.12) at the uniform state. A further evaluation of (2.12) at $Z=0$ then yields two algebraic equations for $\lambda_{1}(0)$ and $\lambda_{2}(0)$, which we write symbolically as

$$
f\left(\lambda_{1}(0), \lambda_{2}(0), \lambda_{1 \infty}, \lambda_{2 \infty}\right)=0, \quad g\left(\lambda_{1}(0), \lambda_{2}(0), \lambda_{1 \infty}, \lambda_{2 \infty}\right)=0,
$$

where $f$ and $g$ are known functions. For each set of $\left(\lambda_{1 \infty}, \lambda_{2 \infty}\right)$, these two equations can be solved to find $\lambda_{1}(0)\left(\neq \lambda_{1 \infty}\right)$ and $\lambda_{2}(0)\left(\neq \lambda_{2 \infty}\right)$, assuming that such a non-trivial solution exists. These values together with $\phi(0)=0$ can then be used as the initial conditions when (2.10) is integrated to find the bulging solution. For the case of fixed $F$, both $\lambda_{2 \infty}$ and $P$ can be expressed in terms of $\lambda_{1 \infty}$ (it is more convenient to use $\lambda_{1 \infty}$ as the control parameter since it decreases monotonically during the entire inflation process). As a result calculation of the bulging solution is straightforward to implement on Mathematica (Wolfram Research, Inc. 2015); see Pearce and Fu (2010). In Figure 1(a), we have shown the dependence of $\lambda_{1}(0)$ on $\lambda_{1 \infty}$ when $F=0$. The intersection of the non-trivial branch with the trivial branch $\lambda_{1}(0)-\lambda_{1 \infty}=0$ gives the bifurcation value $\lambda_{1 \mathrm{cr}}$ of $\lambda_{1 \infty}$. As $\lambda_{1 \infty}$ is reduced from $\lambda_{1 \mathrm{cr}}$, the radius $r(0)$ at the crown of the bulge grows until it has almost reached its maximum 
(corresponding to point $\mathrm{K}$ in the figure). The final stage of radius growth towards its maximum is accompanied by rapid propagation of the bulged section in the axial direction. Theoretically, the maximum of $r(0)$ can only be reached when the bulged section has become infinitely long. When $r(0)$ reaches its maximum, we have $r^{\prime \prime}(0)=0$ as well as $r^{\prime}(0)=0$ so that the state at the crown becomes a second fixed point of the dynamical system (2.10). The associated bulging solution becomes a "hat" solution represented by the joining of two kink solutions which are mirror images of each other. A kink solution in the dynamical systems theory is a heteroclinic orbit that approaches two different fixed points of the the dynamical system as $Z \rightarrow \pm \infty$. The state at $Z=0$ being also a fixed point of (2.10) is represented by the fact that

$$
\frac{w_{1}\left(\lambda_{1 \infty}, \lambda_{2 \infty}\right)}{\lambda_{1 \infty} \lambda_{2 \infty}}=\frac{w_{1}\left(\lambda_{1}(0), \lambda_{2}(0)\right)}{\lambda_{1}(0) \lambda_{2}(0)}
$$

which, in conjunction with (2.13), can be used to determine the values of $\lambda_{1 \infty}, \lambda_{1}(0)$, and $\lambda_{2}(0)$, and hence the propagation pressure $\left(\lambda_{2 \infty}\right.$ is determined by the closed-end condition). Geometrically, this propagation pressure satisfies the equal-area rule (Chater and Hutchinson 1984). At any stage of the inflation process, the bulging solution can be obtained by a single integration of the dynamical system (2.10) since the starting values of the dependent variables at $Z=0$ are already determined as explained above.

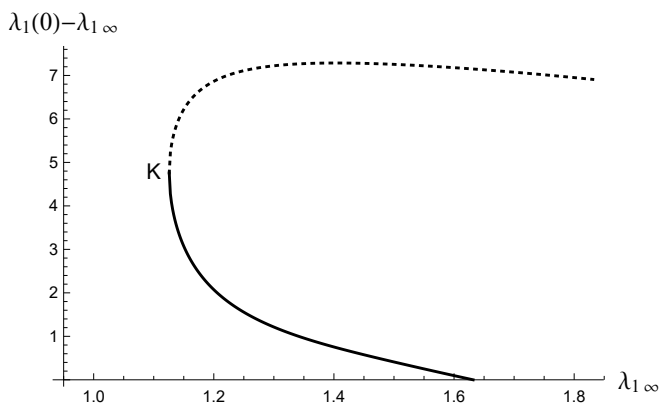

(a)

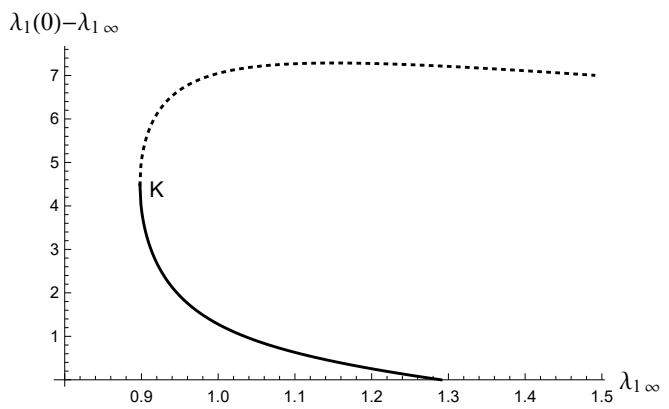

(b)

Figure 1: Solutions of (2.13) when the strain-energy function is given by the Gent-Gent material model (3.16) with $J_{m}=88.43, \mu=0.2853, C_{2}=0.1898$. (a) The case of fixed axial force $F=0$ for which $\lambda_{1 \mathrm{cr}}$ is 1.6318 , and the turning point $\mathrm{K}$ corresponds to $\lambda_{1 \infty}=1.1258, \lambda_{2 \infty}=1.0038, \lambda_{1}(0)=5.8834, \lambda_{2}(0)=4.1241$. As $\lambda_{1 \infty}$ decreases from $\lambda_{1 \text { cr }}$ to 1.1258 at $\mathrm{K}$, the $\lambda_{2 \infty}$ decreases monotonically from 1.0966 to 1.0038 . (b) The case of fixed axial stretch $\lambda_{2 \infty}=1.6$ for which $\lambda_{1 \mathrm{cr}}$ is 1.2904 and the turning point corresponds to $\lambda_{1 \infty}=0.8980, \lambda_{1}(0)=5.3861, \lambda_{2}(0)=4.1635$. Note that in this imaginary case one end of the tube must be extended to maintain the constant axial stretch there.

In the other case when the tube is first stretched and then the ends are fixed during subsequent inflation, $\lambda_{2 \infty}$ and $P$ can no longer be expressed in terms of $\lambda_{1 \infty}$ alone. As a result, the starting values of $\lambda_{1}(0)$ and $\lambda_{2}(0)$ cannot be determined beforehand for each $\lambda_{1 \infty}$, and a shooting procedure needs to be employed in integrating the governing equations (2.10). However, the assumption that the tube is sufficiently long and the two principal stretches near each end are uniform still enables us to provide a semi-analytical, and sufficiently 
accurate, description of the entire inflation process. In our experiments, both ends of the tube are completely fixed, and a boundary layer exists near each end where the deformation is non-uniform. Such boundary layer effects are neglected in our calculations, and the error associated with this approximation will be assessed later with the aid of fully numerical simulations using Abaqus.

We denote the initial axial pre-stretch by $\lambda_{z 0}$. As soon as the bulge begins to grow in diameter after its initiation, the axial stretch $\lambda_{2 \infty}$ near the ends of the tube will decrease. This is manifested through the fact that if the initial stretch $\lambda_{z 0}$ or tube length $L$ is not large enough, the tube may very quickly experience Euler-type buckling (Goncalves et al 2008). Thus, $\lambda_{2 \infty}$ varies as the bulge grows and its value is determined by the fixed-length condition

$$
\int_{0}^{L} z^{\prime}(Z) d Z=\int_{0}^{L} \lambda_{2} \cos \phi d Z=\lambda_{z 0} L .
$$

A shooting procedure with (2.15) as the target condition is therefore required to determine the bulging solutions. A good starting point for this shooting procedure is to take $\lambda_{2 \infty}=\lambda_{z 0}$, since in the limit $L \rightarrow \infty$, the reduction in $\lambda_{2 \infty}$ due to bulge formation should become negligible and $\lambda_{2 \infty} \rightarrow \lambda_{z 0}$. To avoid the numerical difficulty that the bulging solution may start to grow after decaying for a range of $Z$ values, we only integrate $(2.10)$ from $Z=0$ up to a value of $Z, Z^{*}$ say, where $r\left(Z^{*}\right)-\lambda_{1 \infty}$ has become less than $10^{-8}$. For $Z>Z^{*}$, we simply replace $\lambda_{2}$ by $\lambda_{2 \infty}$ and $\phi$ by 0 in the evaluation of the second integral in (2.15).

We first explain the determination of the propagation pressure for each fixed $\lambda_{2 \infty}$ without having to satisfy the fixed-length condition (2.15). This is an imaginary situation in which the tube is allowed to extend in the axial direction in order to maintain the constant $\lambda_{2 \infty}$ near the two ends, but it is a building block for our subsequent calculations. With $\lambda_{1 \infty}$ as the control parameter and $\lambda_{2 \infty}$ fixed, the two equations $(2.13)$ can be solved to find $\lambda_{1}(0)$ and $\lambda_{2}(0)$, and a typical solution with $\lambda_{2 \infty}=1.6$ is shown in Figure 1(b). As in the case of fixed $F$, the intersection of the two solution branches (one of them is the horizontal axis) defines the bifurcation point, and at point $K$ the extra condition (2.14) is also satisfied, and can be used to determine the associated value of $\lambda_{1 \infty}$, which we denote by $\lambda_{1 k}$. The latter quantity is obviously a function of $\lambda_{2 \infty}$. We will eventually need to find the value of $\lambda_{2 \infty}$ at which the fixed-length condition (2.15) is also satisfied.

We note that the values of $\lambda_{1}(0)$ and $\lambda_{2}(0)$ at $K$ cannot be used as initial conditions for (2.10) in order to obtain the kink solution since the state associated with $K$ is a fixed point of the dynamical system (2.10). Neither is it realistic to determine the kink solution exactly because such a solution is defined over an infinite domain. A good approximate representation of the kink solution is the bulging solution corresponding to $\lambda_{1 \infty}=(1+\delta) \lambda_{1 k}$, where $\delta$ is a sufficiently small and positive number. From now on we shall refer to this solution as the near-kink solution, and the associated state as the near-kink state. The relative error in approximating the maximum value of $r(0)$ is of the order $\delta^{1 / 2}$ since the curve near $K$ is locally parabolic. We take $\delta=10^{-8}$ in our calculations. A typical such near-kink solution is shown in Figure 2 using the same scale for both axes in order show the actual appearance of 


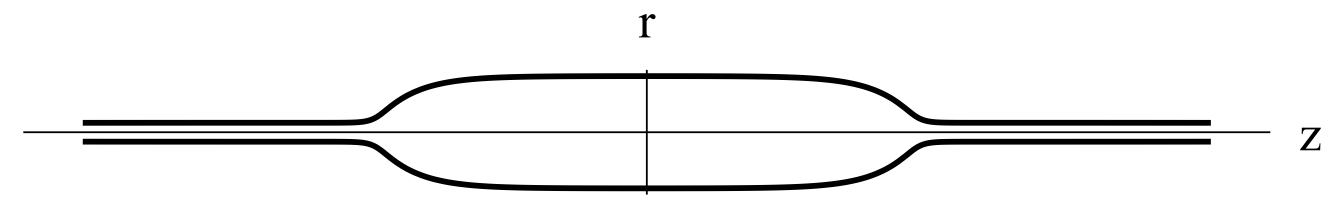

Figure 2: A near-kink solution of (2.10) when the strain-energy function is given by the Gent-Gent material model (3.16) with $J_{m}=89.6, \mu=0.285, C_{2}=0.19, \lambda_{1 \infty}=(1+\delta) \lambda_{1 k}, \lambda_{2 \infty}=1.5$, and $L=30$. With the same scale used for both axes, it shows the actual appearance of the tube in the current configuration.

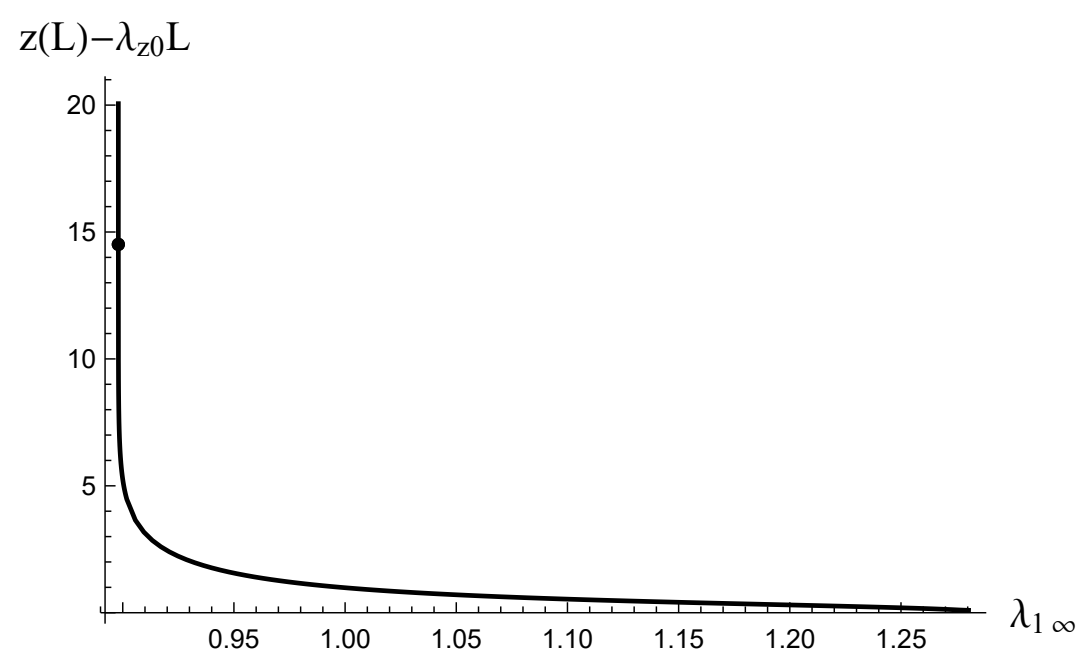

Figure 3: Dependence of end extension on $\lambda_{1 \infty}$, showing that it tends to infinity in the limit $\lambda_{1 \infty} \rightarrow \lambda_{1 k}$, that is $\delta \rightarrow 0$. The dot on the curve corresponds to $\delta=10^{-8}$, and the associated pressure value is taken to be the (approximate) propagation pressure.

the bulged tube. We note that the middle section in Figure 2 would become infinitely wide in the limit $\delta \rightarrow 0$; see Figure 3. This is because the exact kink solution is defined over an infinite interval.

With the above procedure for finding the near-kink solution for each fixed value of $\lambda_{2 \infty}$ established, we may now consider the case when the tube length is fixed, that is when (2.15) is imposed. The actual $\lambda_{2 \infty}$ is expected to be smaller than $\lambda_{z 0}$, and is determined by iteration with the use of the target condition (2.15). In Figure 4, we have shown the dependence of $\lambda_{2 \infty}$ and the associated propagation pressure on $L$ when the near-kink state is reached. It is seen that both $\lambda_{2 \infty}$ and the associated propagation pressure approach their respective limits as $L \rightarrow \infty$ although quite slowly .

We next compute the bulging solutions before the kink state is reached in order to understand the variation of the inflation pressure. It is found that as the bulge amplitude $r(0)$ grows, the $\lambda_{2 \infty}$ decreases monotonically, but the variation of $\lambda_{1 \infty}$ is not monotonic. This is in contrast with the case of fixed $F$ for which both $\lambda_{1 \infty}$ and $\lambda_{2 \infty}$ decrease monotonically as the bulge grows. Thus, it is convenient to decrease $\lambda_{2 \infty}$ in small steps, and at each step we determine the associated $\lambda_{1 \infty}, \lambda_{1}(0)$, and $\lambda_{2}(0)$ by satisfying the three conditions in (2.15) and (2.13). More precisely, for each $\lambda_{2 \infty} \in\left(\lambda_{z k}, \lambda_{z 0}\right)$, where $\lambda_{z k}$ is the value of $\lambda_{2 \infty}$ when 


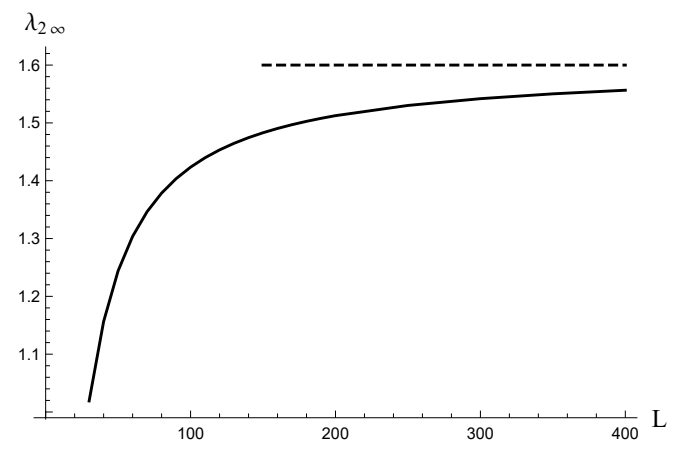

(a)

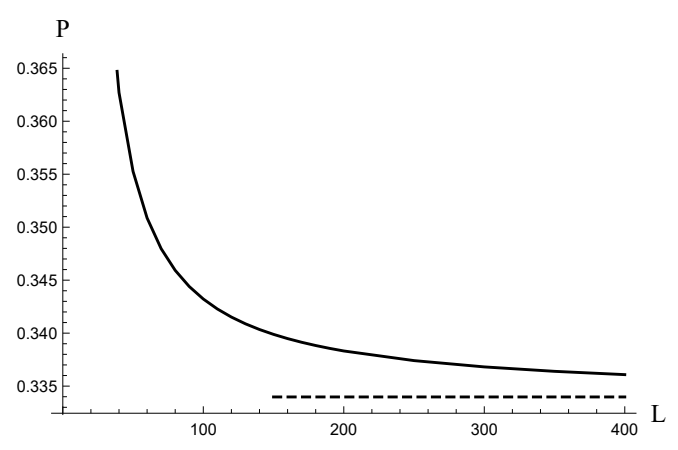

(b)

Figure 4: Dependence of $\lambda_{2 \infty}$ and the associated propagation pressure on $L$ when the strain-energy function is given by the Gent-Gent material model (3.16) with $J_{m}=89.6, \mu=0.285, C_{2}=0.19$. The dashed line is the theoretical result in the limit $L \rightarrow \infty$. When $L=400$, the actual values of $\lambda_{2 \infty}$ and the associated propagation pressure differ from their asymptotic limits with relative errors equal to $3 \%$ and $0.7 \%$, respectively. The axial stress at the end of the tube becomes negative when $L$ is approximately equal to 30, meaning that the tube would suffer Euler-type buckling if $L<30$.

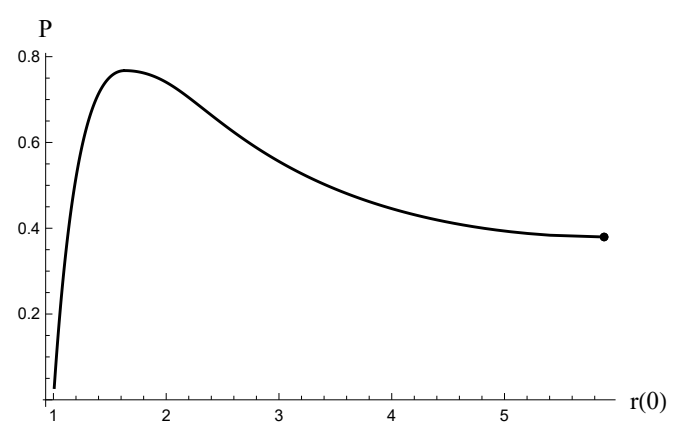

(a)

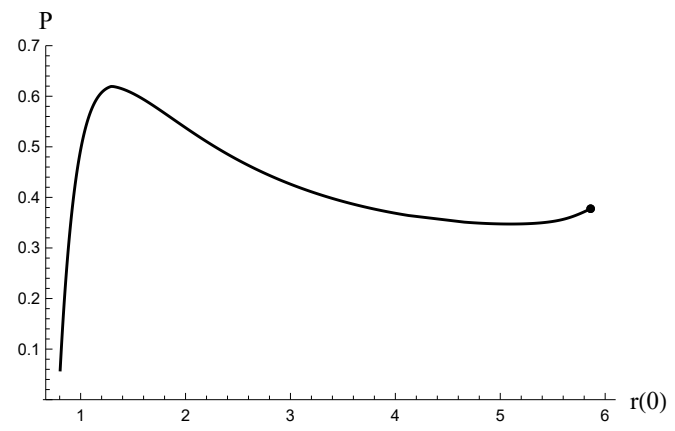

(b)

Figure 5: Dependence of scaled pressure $P$ on the bulge amplitude $r(0)$ as the bulge grows. (a) The case of fixed $F=0$. (b) The case of fixed length $\lambda_{z 0} L$ with $\lambda_{z 0}=1.6, R_{m}=3.75 \mathrm{~mm}, H=0.4, L=30$. The dots in (a) and (b) correspond to the kink state and near-kink state, respectively.

the near-kink state is first reached, we iterate on $\lambda_{1 \infty}$ so as to satisfy the fixed end condition (2.15), and for each set of $\left(\lambda_{1 \infty}, \lambda_{2 \infty}\right)$, the bulge amplitude is determined by solving the two conditions (2.13). In Figure 5(b), we have shown the dependence of pressure on the bulge amplitude up to the point when the near-kink state is reached. In this plot the part before the pressure maximum corresponds to uniform inflation, and the part after the pressure maximum corresponds to the bulging solution. As a comparison, we have also shown in Figure 5(a) the counterpart of Figure 5(b) when $F$ is fixed, the segment corresponding to the bulging solutions being obtained by following the solid line in Figure 1(a). In the two plots, the dots corresponds to the exact kink state and near-kink state, respectively.

Figures 5(a) and (b) are obviously different. In the case of fixed axial force, the pressure decreases monotonically, and when the pressure becomes sufficiently close to the Maxwell value, the bulge will rapidly propagate in the axial direction. In contrast, in the case of fixed 
tube length, the pressure first decreases monotonically to a minimum before it increases monotonically to reach its value corresponding to the near-kink state. For the case considered in Figure 5(b), the pressure maximum and minimum are found to be 0.6194 and 0.3473 , respectively. To validate these results, we have also run a numerical simulation using the software Abaqus for a tube of the same material properties. All of our Abaqus simulations here and hereafter are based on the exact three-dimensional nonlinear elasticity theory. The inner radius, outer radius and half-length $L$ of the tube are assumed to be equal to 3.5, 4 , and 112.5 units, respectively. This corresponds to a scaled $L$ equal to 30 , and a scaled wall thickness $H=\varepsilon=0.13$ so that the tube wall is quite thin. We have considered the following three different types of imperfections to make sure that the bulge will initiate at the central section of the tube $(Z=0)$ : (1) The radial movement at each end is un-restricted but in-plane rotation at four equally spaced points on the outer surface is restricted. This mimics the assumptions behind our semi-analytical computations. We introduce a very small weakening of the material properties by assuming that the value 0.2853 of $\mu_{0}$ given by Table 2 is replaced by 0.2850 for one eighteenth of the total length of the tube around $Z=0$ while the values of $J_{m}$ and $C_{2}$ remain unchanged; (2) We introduce the same material weakening as in (1), but the in-plane movement at both ends is completely restricted. (3) We restrict completely in-plane movement at both ends, but no material weakening is introduced, which mimics the situation in our experiments. The restrictions at the ends serve as imperfections and because of symmetry the bulge will tend to initiate at $Z=0$. It is found that the results from these three different approaches differ from each other by less than $0.2 \%$. Thus, all the subsequent Abaqus simulations are based on the third approach which is the simplest to implement. For the current case, Abaqus yields the values 0.6177 and 0.3480 for the scaled pressure maximum and minimum, respectively, and our semi-analytical results differ from these results by less than $0.3 \%$. We have run simulations for three other tubes with increasingly larger and larger wall thickness but the same scaled half-length 30 . These tubes have inner and outer radii $(A, B)$ given by $(3,4.5),(2.5,5)$, and $(2.25,5.25)$, respectively. The results are summarized in Table 1 . The 3D theory used to compute the initiation pressure refers to the theory of $\mathrm{Fu}$ et al (2016) so that the initiation pressure (i.e. pressure maximum) is determined by (2.5), and a cross signifies the fact that this theory cannot compute the pressure minimum. In addition to confirming the claim in Fu et al (2016) that the membrane theory can be used to accurately predict the initiation pressure for thin and moderately thick rubber tubes, these typical computations also confirm that the current semi-analytical approach is also accurate in predicting the pressure minimum for thin and moderately thick rubber tubes for the intended end conditions, but it under-predicts the pressure minimum by $8 \%$ when the scaled wall thickness becomes as large as 0.67 . 
Table 1: Comparison with numerical simulations using Abaqus

\begin{tabular}{lccc}
\hline & Membrane theory & Abaqus (fixed ends) & 3D theory \\
\hline$P_{\text {cr }}$ with $\varepsilon=0.13$ & 0.6194 & 0.6177 & 0.6199 \\
\hline$P_{\text {min }}$ with $\varepsilon=0.13$ & 0.3473 & 0.3480 & $\mathrm{X}$ \\
\hline$P_{\text {cr }}$ with $\varepsilon=0.40$ & 0.6194 & 0.6220 & 0.6248 \\
\hline$P_{\text {min with } \varepsilon=0.40}$ & 0.3473 & 0.3577 & $\mathrm{X}$ \\
\hline$P_{\text {cr }}$ with $\varepsilon=0.67$ & 0.6194 & 0.6296 & 0.6367 \\
\hline$P_{\text {min }}$ with $\varepsilon=0.67$ & 0.3473 & 0.3779 & $\mathrm{X}$ \\
\hline$P_{\text {cr with } \varepsilon=0.80}$ with $\varepsilon=0.80$ & 0.6194 & 0.6358 & 0.6374 \\
\hline$P_{\text {min }}$ with & 0.3473 & 0.3945 & $\mathrm{X}$ \\
\hline
\end{tabular}

\section{Experimental set-up and material characterization}

A set of latex rubber tubes were purchased from a commercial company together with a large membrane sheet with thickness $0.5 \mathrm{~mm}$. Six groups of tubes are used in the experiments. Tubes in different groups have the same inner diameter $6 \mathrm{~mm}$ but different outer diameters equal to $9,10,11, \cdots, 14 \mathrm{~mm}$, respectively, and are believed to have the same material properties as the membrane sheet provided. Thus, the corresponding thickness/radius ratio $\varepsilon$ for the six tubes is equal to $0.4,0.5,0.59,0.67,0.74,0.8$, respectively. Tubes in the first four groups can be classified as membrane tubes for which the membrane theory should provide a good approximation for the exact 3D theory ( $\mathrm{Fu}$ et al 2016). In particular, for the initiation pressure the relative error should be less than $5 \%$.

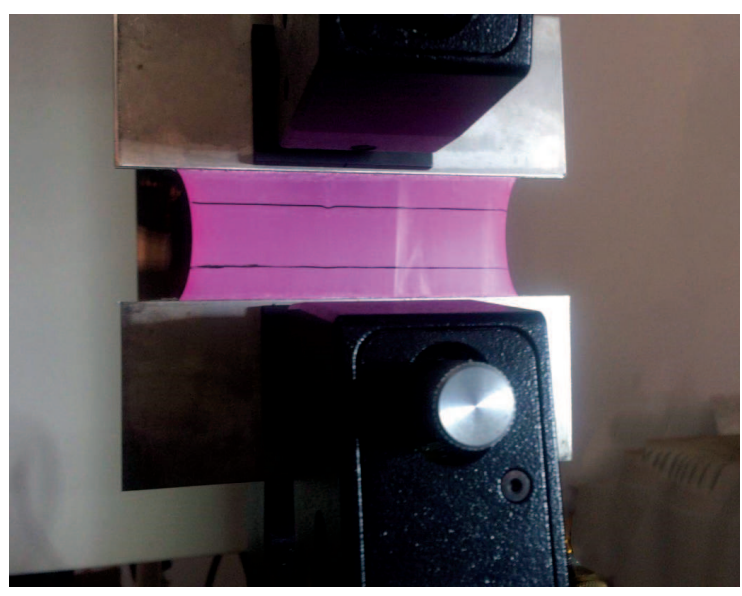

(a)

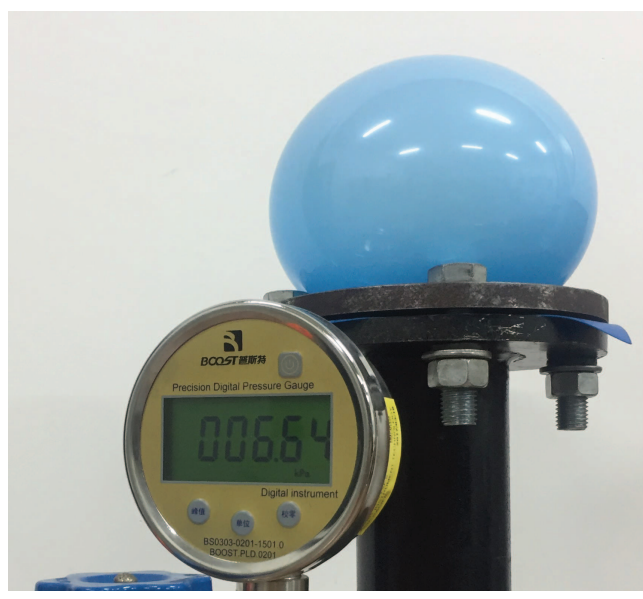

(b)

Figure 6: Final stages of (a) pure shear and (b) biaxial extension.

To characterize the material properties, we use the membrane sheet to prepare samples to conduct uni-axial tension, pure shear, and equi-biaxial extension, following the protocol described by Treloar (1944a). In particular, for uni-axial tension, the effective sample size 
in the dumb bell shaped sample is $6 \mathrm{~mm} \times 33 \mathrm{~mm}$, and the maximum longitudinal stretch reached is 6.5. For pure shear, the effective sample size is $5 \mathrm{~mm} \times 100 \mathrm{~mm}$; the sample is clamped along the long sides by two rigid plates and then the plates are pulled apart (see Figure 6(a)), the maximum stretch reached in the pulling direction being 6.4. For equi-biaxial extension, we inflate a circular plane membrane by using it to seal a cylindrical gas chamber (see Figure 6(b)). The effective test area is of diameter $90 \mathrm{~mm}$. It is well-known that as the pressure in the gas chamber is increased, the membrane will be inflated into a spherical shell approximately, and near the pole the deformation can be viewed to be equi-biaxial (Treloar 1944b), the maximum stretch reached being 5.5. All samples are pre-stretched or pre-inflated a number of times to minimize Mullins' effects following the standard practice, and all experiments were conducted at a room temperature of $20 \pm 1^{\circ}$ C. All stretches are obtained by high-precision image processing. More details on the equi-biaxial experiments can be found in Zhou et al (2018).

Five sets of valid results were obtained for each type of deformation. In view of the comparative studies presented in Pucci and Saccomandi (2002) and Ogden et al (2004), we fit our experimental data to the Gent-Gent material model given by

$$
W=-\frac{\mu_{0}}{2} J_{m} \ln \left(1-\frac{I_{1}-3}{J_{m}}\right)+C_{2} \ln \left(\frac{I_{2}}{3}\right),
$$

where

$$
I_{1}=\lambda_{1}^{2}+\lambda_{2}^{2}+\lambda_{3}^{2}, \quad I_{2}=\lambda_{1}^{2} \lambda_{2}^{2}+\lambda_{1}^{2} \lambda_{3}^{2}+\lambda_{2}^{2} \lambda_{3}^{2},
$$

and $\mu_{0}, J_{m}, C_{2}$ are constants. The ground state shear modulus $\mu$ associated with this model is given by

$$
\mu=\mu_{0}+\frac{2}{3} C_{2}
$$

In particular, we chose this model on the grounds that there exists a unique solution in the data fitting, and it overcomes the shortcoming that the original Gent model cannot accurately capture the behaviour at moderate values of stretch. Moderate values of stretch are precisely where localized bulging initiates. Recent studies by Mangan and Destrade (2015) and Zhou et al (2018) have provided more supporting evidence that the Gent-Gent model is superior to the original Gent model. 


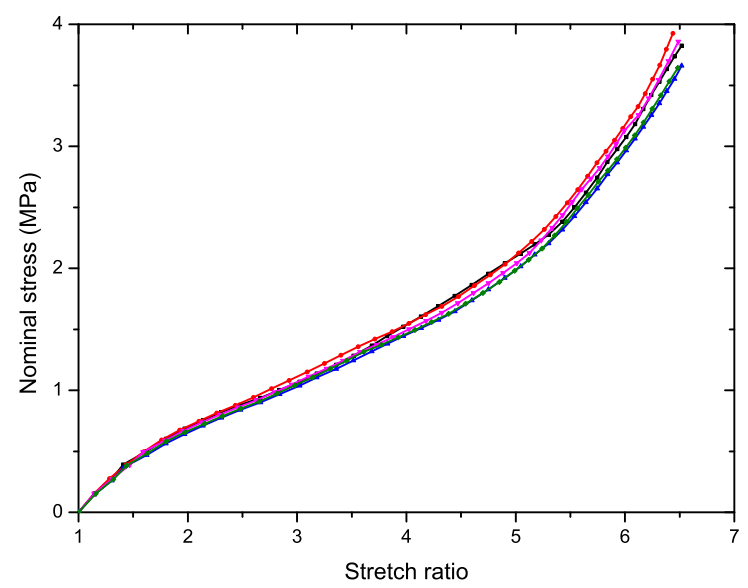

(a)

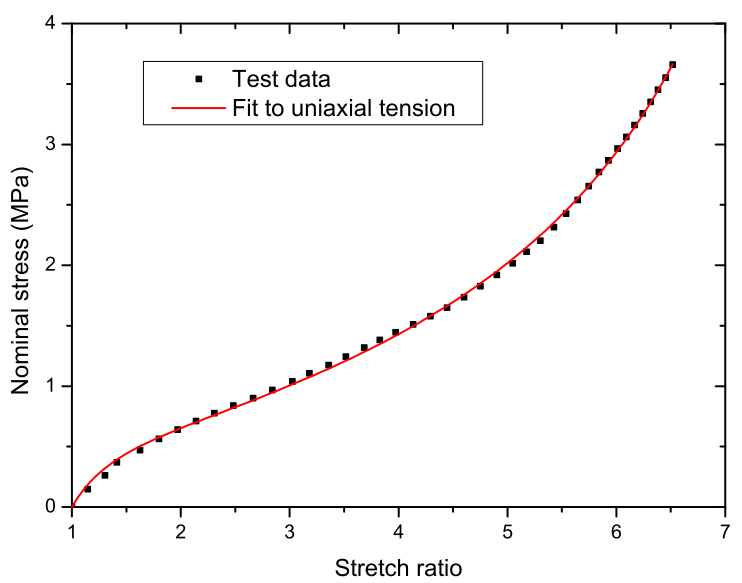

(b)

Figure 7: (a) Five sets of experimental data on uniaxial tension, and (b) one of them fitted to the Gent-Gent model

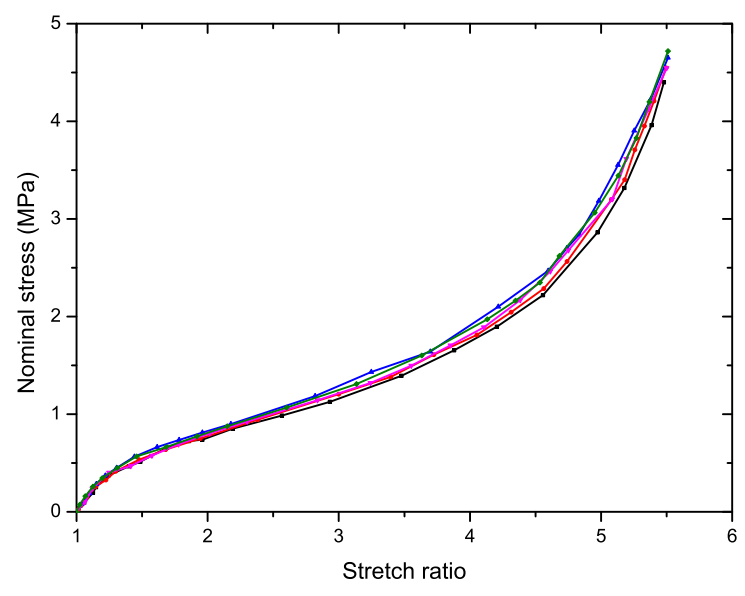

(a)

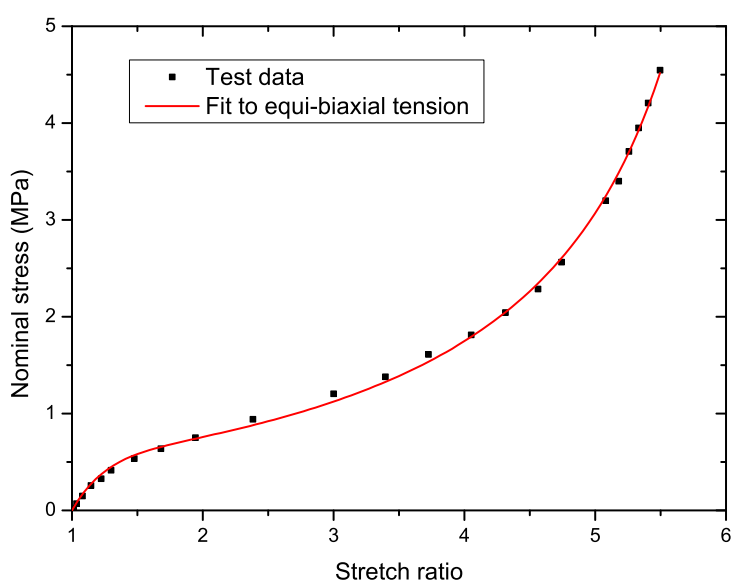

(b)

Figure 8: (a) Five sets of experimental data on equi-biaxial tension, and (b) one of them fitted to the Gent-Gent model 
Table 2: Averaged values of material constants from data fitting

\begin{tabular}{lcccc}
\hline & $J_{m}$ & $\mu_{0}(\mathrm{MPa})$ & $C_{2}(\mathrm{MPa})$ & $\mu(\mathrm{MPa})$ \\
\hline Uni-axial tension & 79.65 & 0.2903 & 0.3802 & 0.5438 \\
\hline Pure shear & 76.22 & 0.2718 & 0.2280 & 0.4238 \\
\hline Equi-biaxial extension & 88.43 & 0.2853 & 0.1898 & 0.4118 \\
\hline
\end{tabular}

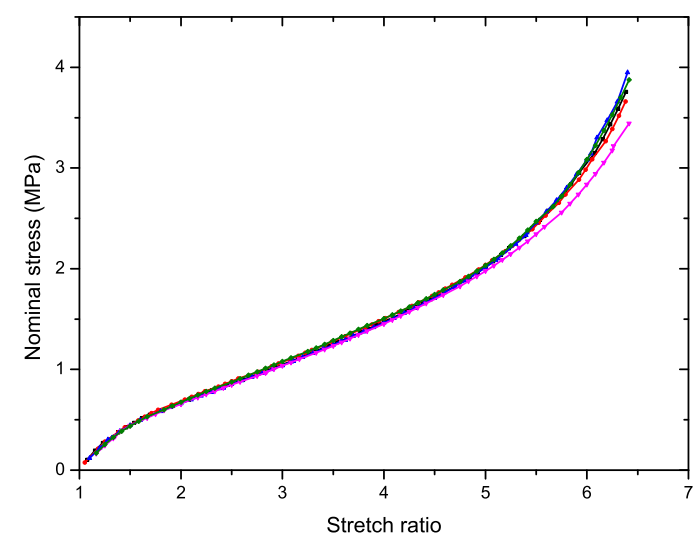

(a)

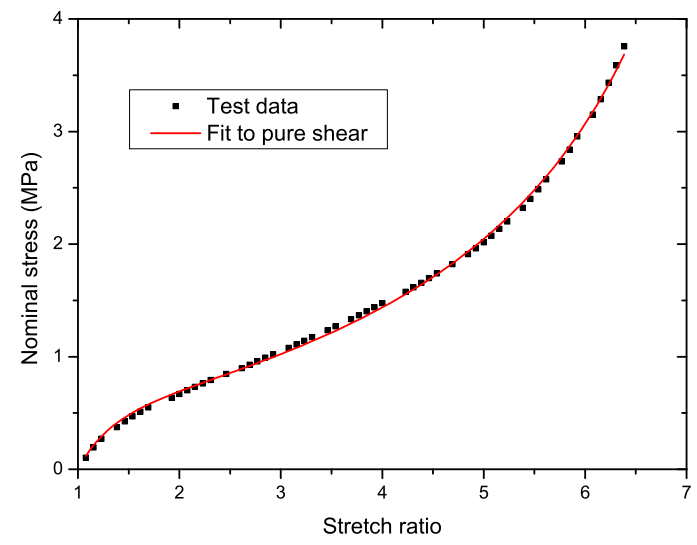

(b)

Figure 9: (a) Five sets of experimental data on pure shear, and (b) one of them fitted to the Gent-Gent model

The experimental data giving the nominal stress against the corresponding stretch are shown in Figures 7-9. In the right part of each figure, we show a typical result of fitting the above Gent-Gent model to one set of experimental data. The averaged values of the material constants are presented in Table 2 . In view of the fact that the equi-biaxial extension test is closest to the deformation of localized bulging, we shall use the averaged material constants based on equi-biaxial extension to produce theoretical predictions; that is, in the rest of this paper we shall take $J_{m}=88.43, \mu_{0}=0.2853(\mathrm{MPa}), C_{2}=0.1898(\mathrm{MPa})$. In Figure 10, we compare the theoretical predictions based on this set of material constants with a typical set of experimental data for uni-axial tension and pure shear. The maximum error in each case is around $12 \%$, which is typical for such cross comparisons; see, for instance, Ogden et al (2004). 


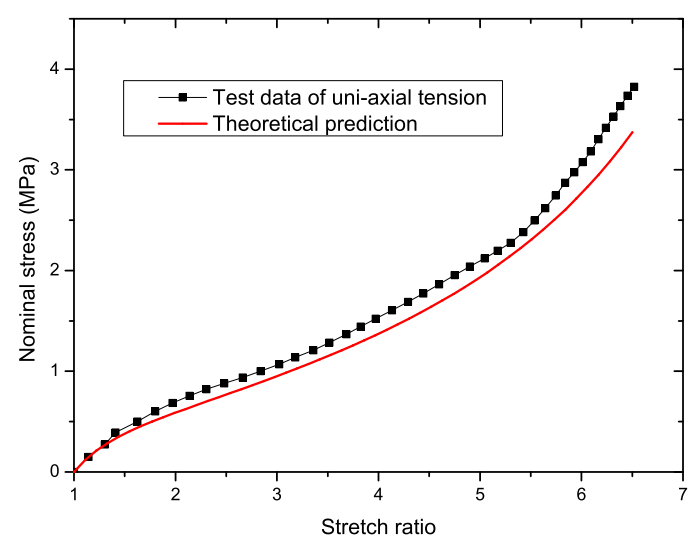

(a)

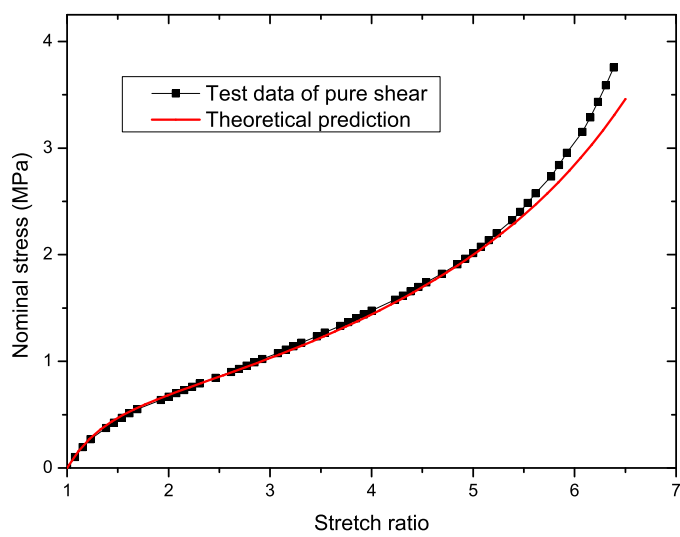

(b)

Figure 10: Theoretical prediction (solid line) based on $J_{m}=88.43, \mu_{0}=0.2853, C_{2}=0.1898$ for (a) uni-axial extension, and (b) pure shear. The dots are a representative set of experimental results.

\section{Comparison of analytical and experimental results}

We use an air pump to inflate the tubes, a high-speed camera to record the images, and a synchronous measuring system to record the internal pressure in all of our experiments. In the case of fixed force, the camera records 200 frames per second, and in the case of fixed stretch it is 100 frames per second (the sample rate is lowered to reduce data size). Since it is not possible to measure the inner radius of the tube during inflation, the radius referred to in the subsequent discussion is the outer radius. The high contrast between the white tube and the black background enabled MATLAB's image processing tools to be used to quantify the outer diameter for every captured image. In the image processing, each row of pixels was scanned to obtain the first and last white pixel locations. The largest difference between pixel locations was taken as the bulge diameter and converted from pixels to mm. To remove Mullins' effect, each tube is inflated three times first and data are only collected at the fourth inflation. It is found that the initiation pressure and the propagation pressure or minimum pressure will both decrease further, although very slowly, as the number of inflations is increased. Typically, the initiation pressure at the 25 th inflation is around $9 \%$ lower than its value at the 4 th inflation, whereas with the propagation or minimum pressure, the corresponding reduction is around $6 \%$. We have also checked whether it will make any difference if the tube is axially stretched a number of times before the first inflation. It is found that such pre-stretching has a noticeable effect on the first inflation, but has little effect on the fourth inflation. To ensure that localized bulging takes place near the middle section of the tube, we always carry out the first inflation on a longer tube, and then cut the tube to the specified length with the bulged section centred around the middle of the tube. Because of the slight residual deformation left by the first inflation in the bulged area, bulging in subsequent inflations tends to occur in the same area. For each data point, we 
carry out inflation on three identical tubes, and the final value adopted is the average of the three values collected.

Before considering the two loading cases separately, we first make some general observations. In all subsequent comparisons, the theoretical initiation pressure is calculated based on the 3D exact bifurcation condition (2.5). In the case of fixed $F$, the theoretical propagation pressure is calculated with the aid of Maxwell's equal area rule using the pressure given by (2.2). In the case of fixed ends, the theoretical minimum pressure is calculated using the procedure explained in Section 2. All our Abaqus simulations are based on the 3D exact nonlinear elasticity theory. As expected, the initiation pressures given by theory and Abaqus are graphically indistinguishable for all cases considered. This is also true for the propagation pressure in the case of fixed $F$. However, for the minimum pressure in the case of fixed ends there is some discrepancy between theory and Abaqus due to the fact that the theoretical minimum pressure is based on the membrane theory and hence tends to underpredict the actual value.

\subsection{The case of fixed $F$}

We first present a typical set of experimental results corresponding to the case when $R_{m}=3.75 \mathrm{~mm}$ (corresponding to inner radius $3 \mathrm{~mm}$, outer radius $4.5 \mathrm{~mm}, \varepsilon=0.4$ ), $2 L=60$, and $F=0.2785$ (recall that $L$ and $F$ have been non-dimensionalized). The nine marked points in each of the Figures 12-14 correspond to the nine configurations in Figure 11. Figure 12 shows that the outer radius approaches its maximum value much more slowly than the pressure approaches its Maxwell value (i.e. the propagation pressure). This can be explained by the fact that near the kink point in Figure 1 the $\lambda_{1 \infty}$, and hence the pressure, are each a parabolic function of the bulge amplitude. The inflation pressure $P$ decreases monotonically towards its Maxwell value, but will never reach the Maxwell value because, as remarked earlier, this can only be achieved when the bulge has become infinitely long. This is consistent with the theoretical prediction noted in the previous section. It can also be seen in Figure 12 that the pressure drops from its maximum to 0.45 in 7 recording frames (each thick dot on the curve represents a recording), that is in about $7 / 200$ seconds. This demonstrates the fact that the initial stage of bulge initiation and growth is unstable even under mass control (Fu and Xie 2010). In Figure 13, we have also marked our theoretical predictions for the initiation and propagation pressures. The experimental value 0.65 for the initiation pressure is about $11 \%$ lower than the theoretical prediction 0.73 , whereas for the propagation pressure the experimental and theoretical values are 0.36 and 0.38 , respectively, the relative difference being $5.3 \%$. This is consistent with the theoretical prediction that the initiation pressure is sensitive to imperfections whereas the propagation pressure is not. 


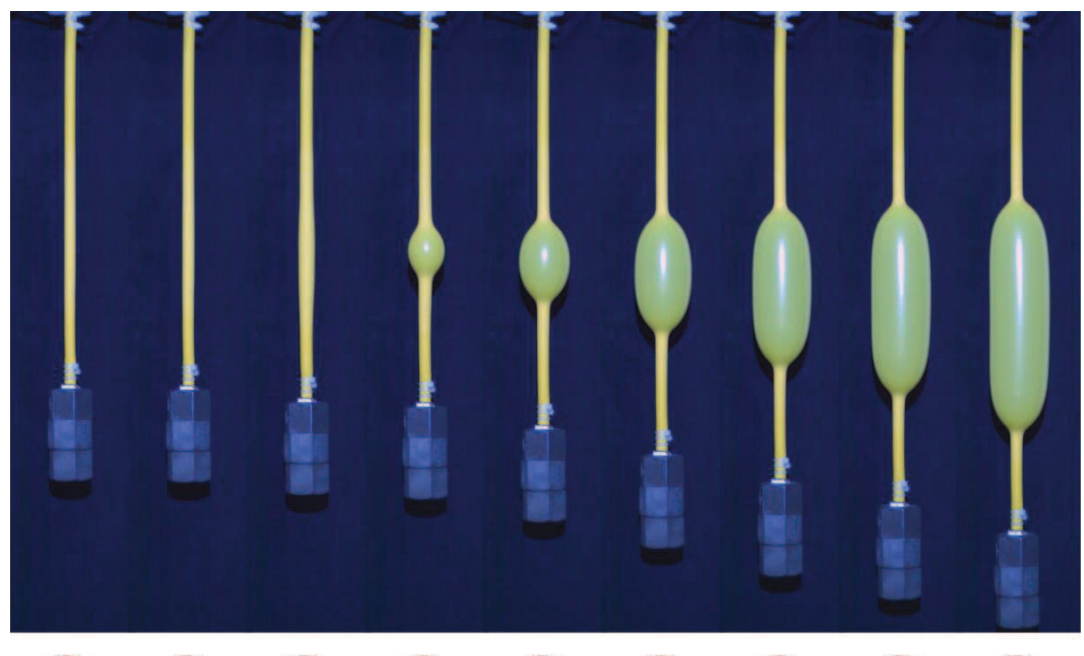
(1)
(2)
(3) (4)
(5)
(6)
(7)
(8)
(9)

Figure 11: A representative set of configurations of a tube with inner diameter $6 \mathrm{~mm}$, outer diameter $9 \mathrm{~mm}$, length $225 \mathrm{~mm}$ (so that $H=0.4, L=30$ ), in the case of fixed $F=0.2785$.

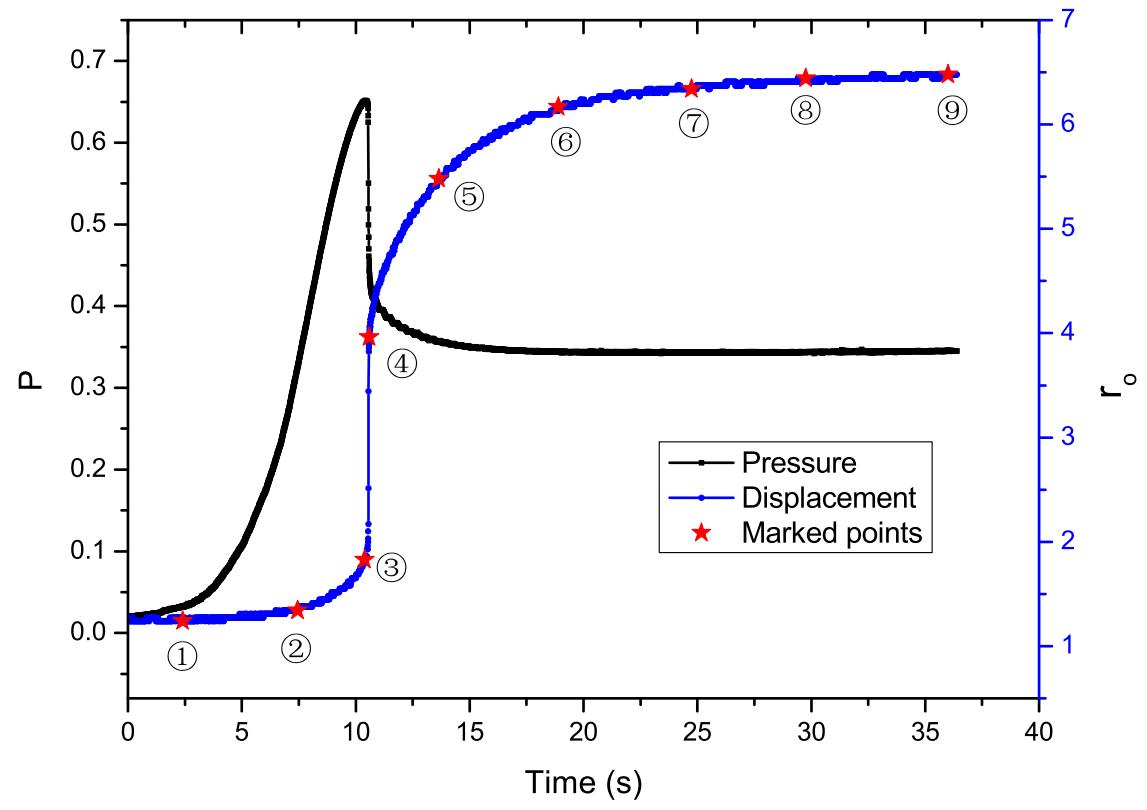

Figure 12: Evolution of pressure and bulge amplitude with time for the same tube as in Figure 11. The nine marked points correspond to the nine configurations in Figure 11. 


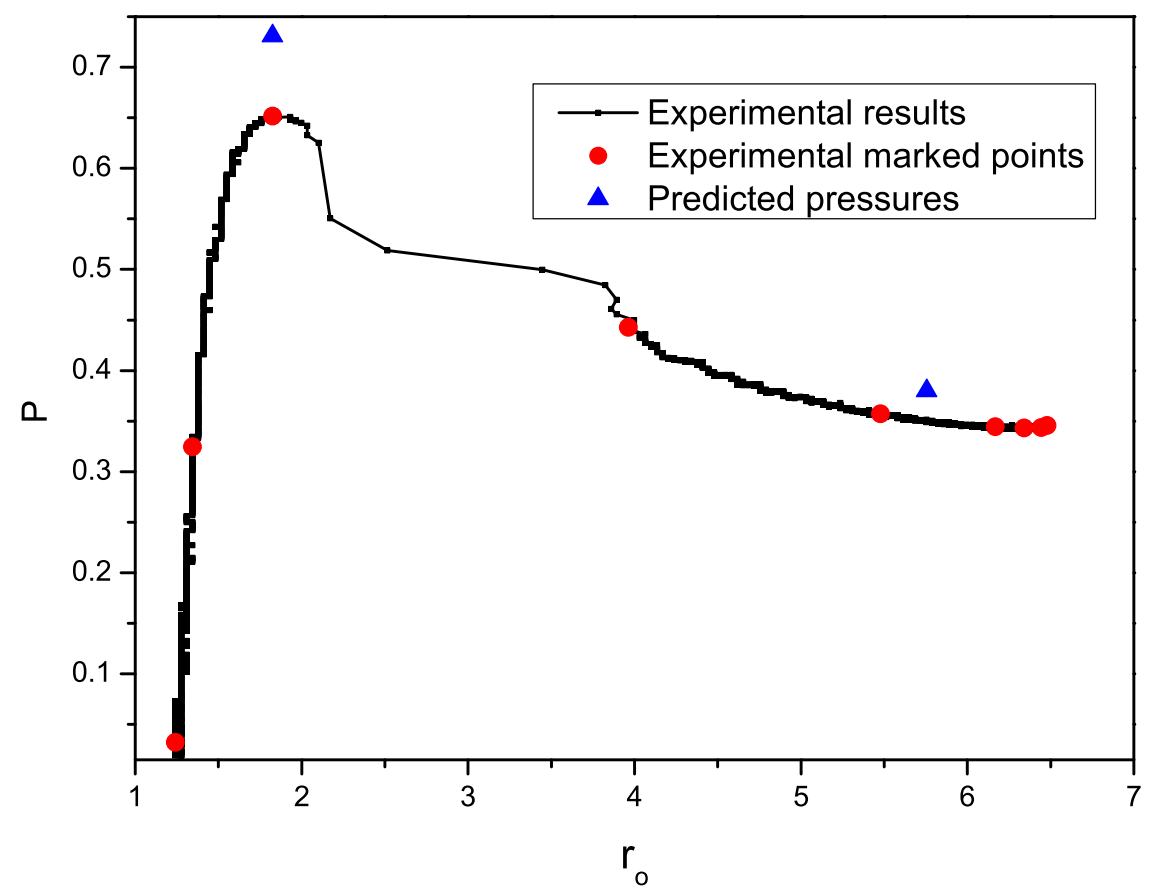

Figure 13: Dependence of pressure on the bulge amplitude. The $r_{r m o}$ in the horizontal axis label is the outer radius of the tube.

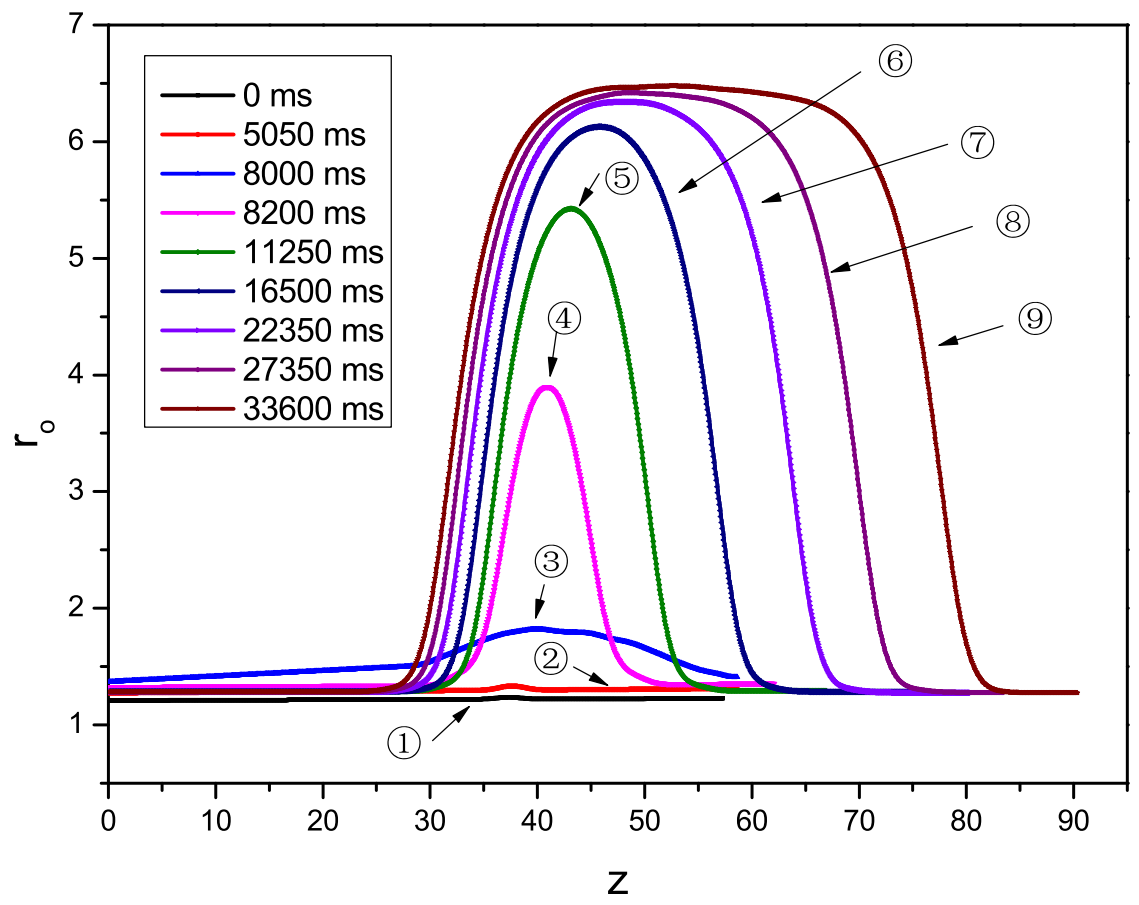

Figure 14: Evolution of bulge profile. The nine profiles correspond to the nine configurations in Figure 11.

The next three figures show the effects of axial force, wall thickness and tube length on the initiation and propagation pressures. Figure 15 shows that as the axial force $F$ is increased, 
the initiation and propagation pressures measured experimentally follow the same trend as the theoretical predictions, both decreasing monotonically. For the initiation pressure the experimental value is consistently between 10-19\% lower than the theoretical prediction, whereas for the propagation pressure there is good agreement between the experimental results and theoretical predictions, showing again the imperfection sensitivity of the former and the robustness of the latter. Figure 16 displays the dependence of the two pressures on the wall thickness, and it is seen that the two pressures are increasing functions of $H$. It is seen that the gap between the experimental and theoretical values for the initiation pressure is getting smaller as the wall thickness is increased. This seems to indicate that the initiation pressure is more sensitive to imperfections for thin-walled tubes than for thick-walled tubes. It is also seen that for the propagation pressure the experimental value is slightly smaller than the theoretical value for the first two $H$ values, but the relation is reversed for the other four higher $H$ values. Figure 17 shows the dependence of the two pressures on the tube length. It is seen that the two pressures are insensitive to the values of the tube length considered. This can be explained by the fact that the bulging solution is effectively a static solitary wave which has amplitude decaying exponentially towards the two ends of the tube. Finally, we remark that although results corresponding to $F=0.2785, H=0.4, L=30$ are given in both Figures 15 and 17, the experimental results are not necessarily identical since they correspond to different physical tubes. In other words, to produce the results in Figure 17, we have used a fresh set of tubes with different specified lengths.

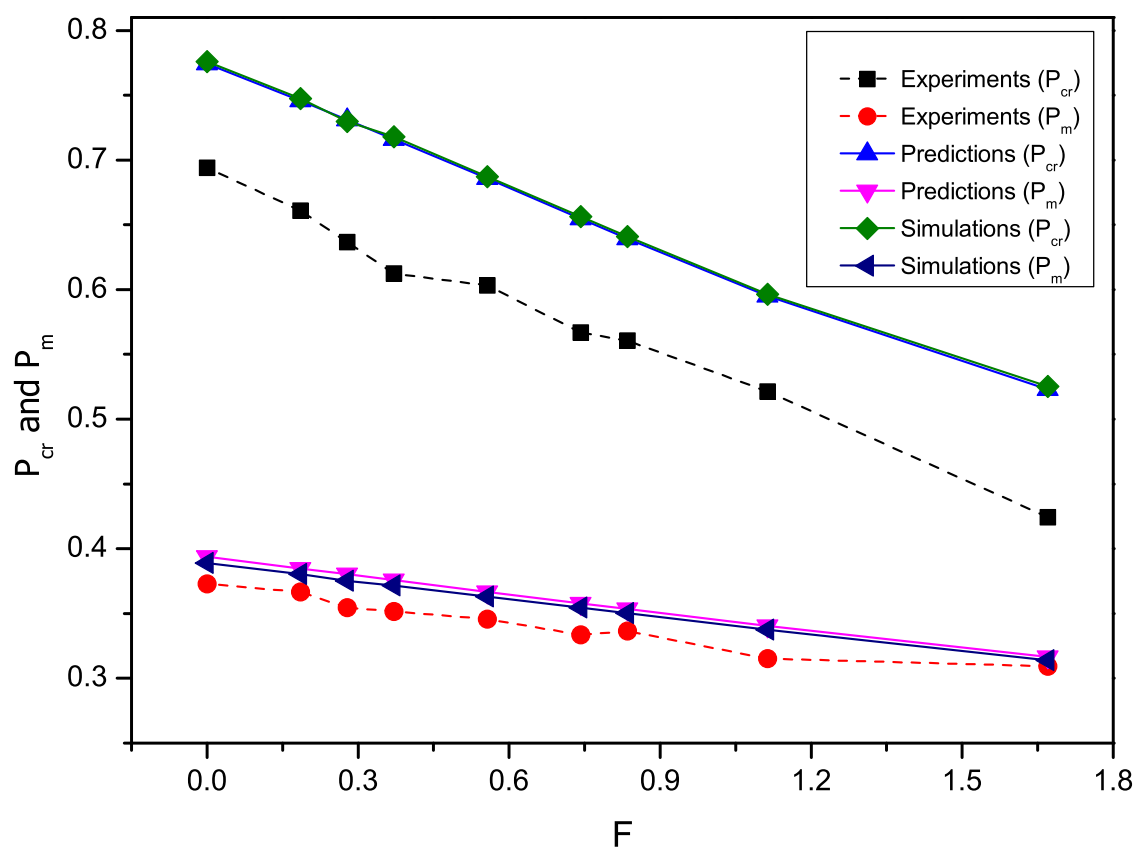

Figure 15: Dependence of the initiation and propagation pressures on $F$ when $H=\varepsilon=0.4, L=30$. 


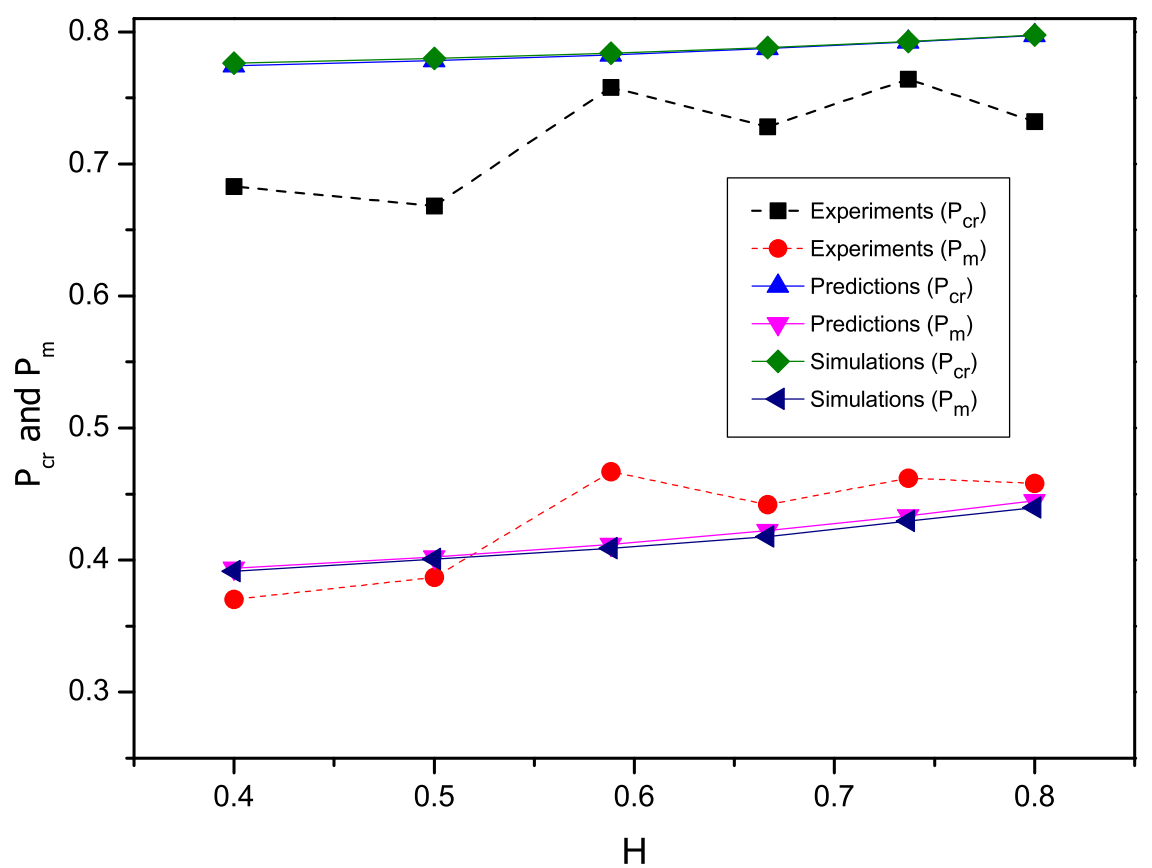

Figure 16: Dependence of the initiation and propagation pressures on wall thickness when $F=0, L=30$.

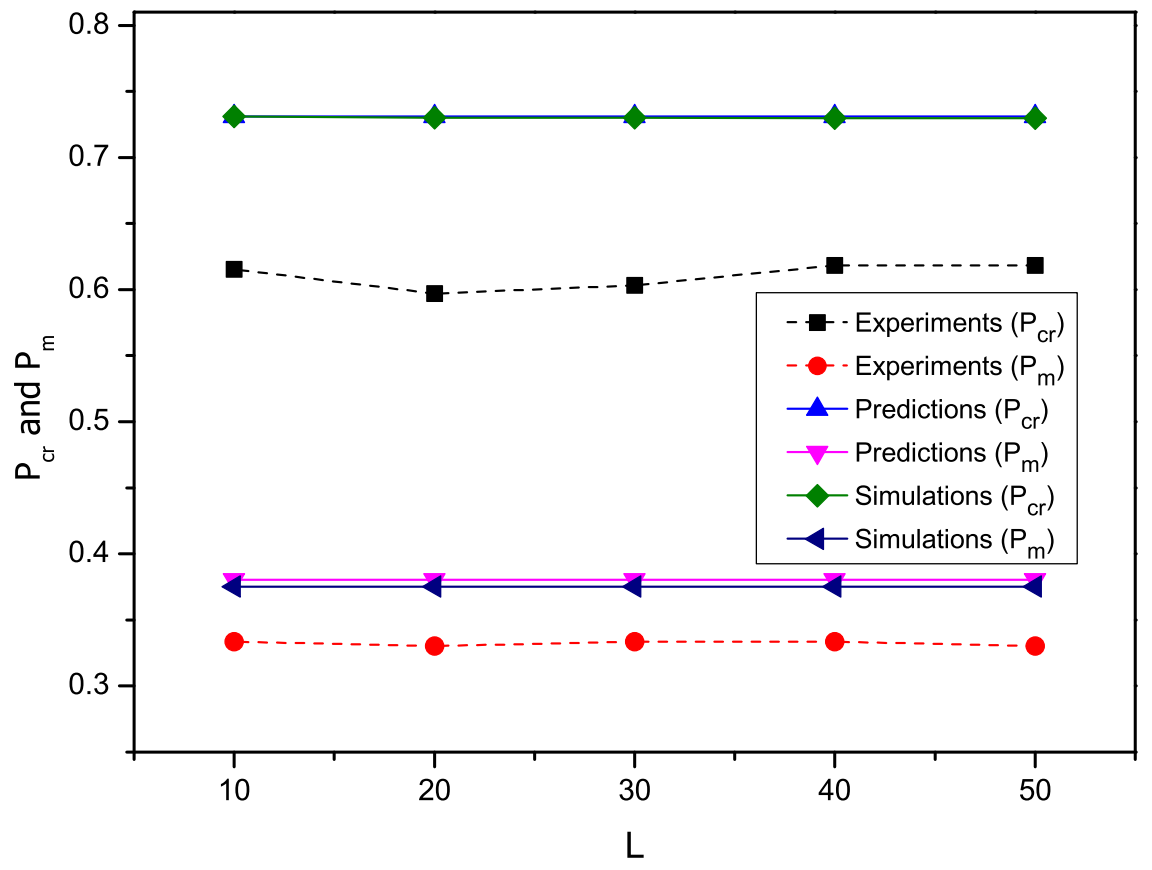

Figure 17: Dependence of the initiation and propagation pressures on the tube length when $H=0.4, F=$ 0.2785

\subsection{The case of fixed ends/length}

In this case the initiation pressure is determined by the same bifurcation condition as in the previous case, but in contrast with the previous case the concept of a propagation 
pressure no longer applies. The pressure would reach a minimum first before it rises again to approach the value corresponding to the near-kink state defined in the previous section. We also note that once the tube has reached the near-kink state, further propagation of the bulge will still be restricted by the fixed ends. As an infinitesimal part $d Z$ of the narrower section is converted into the wider section, it will elongate by $\left(\lambda_{2}(0)-\lambda_{2 \infty}\right) d Z$ which is positive. Since such an elongation is not allowed to happen due to the fixed ends, the narrower section must unload itself further to reach a lower $\lambda_{2 \infty}$ value. This lower value of $\lambda_{2 \infty}$ must be such that the total tube length is shorter than $\lambda_{z 0} L$, the amount of shortening being equal to the extension associated with the conversion of a part of the narrower section into the wider section. Thus, we may alternatively decrease $L$ and compute the associated near-kink state, and hence the value of $r(0)$. Such a calculation shows that the pressure will increase slowly beyond the dot shown in $5(\mathrm{~b})$. This is consistent with what is observed in our experiments to be presented later. This propagation stage is terminated when either the ends are reached or the axial stress at the ends becomes negative, in which case the tube will buckle.

We also remark that under the assumption that Euler-type buckling will occur if the axial stress at the ends becomes negative, the initial stretched length $2 \lambda_{z 0} L$ must be sufficiently large to ensure that the tube will not buckle by the time when the near-kink state is reached. It is found that to satisfy this condition, when $\lambda_{z 0}=1.6$ we must have $L>26$, and when $L=30$ we must have $\lambda_{z 0}>1.5$, both inequalities being approximate. These results are used to guide the design of our experiments.

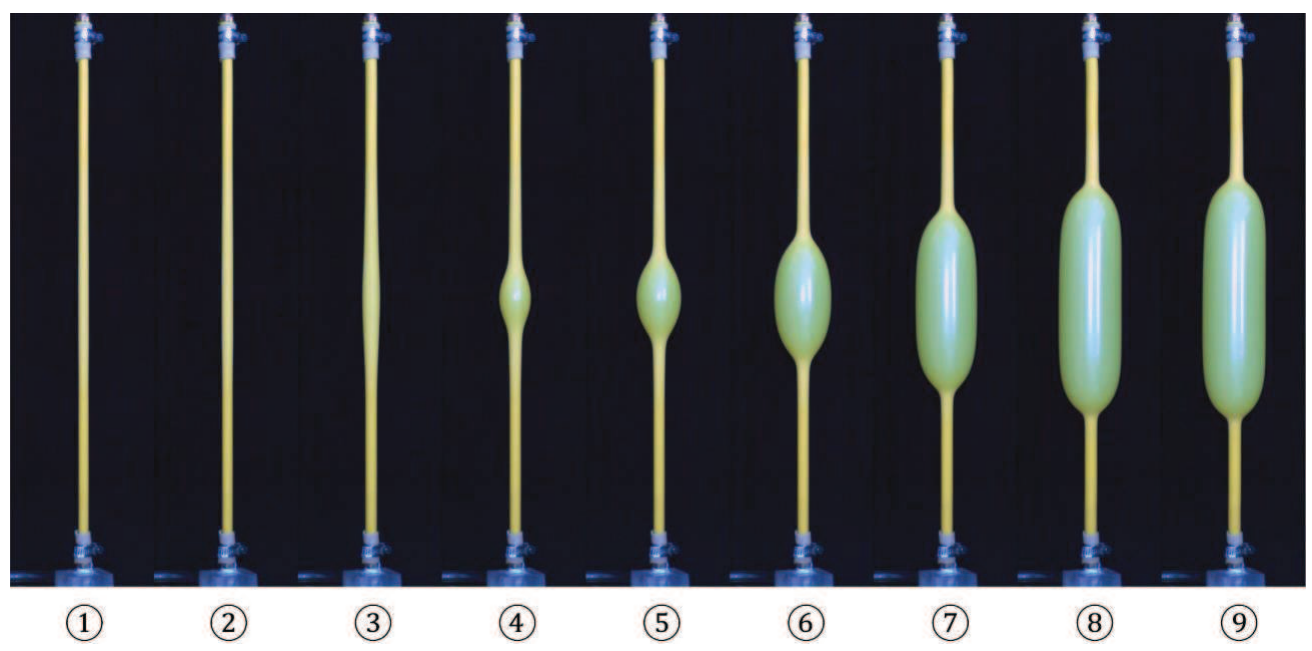

Figure 18: A representative set of configurations of a tube with inner diameter $6 \mathrm{~mm}$, outer diameter $9 \mathrm{~mm}$, length $225 \mathrm{~mm}$ (so that $L=30, H=\varepsilon=0.4$ ), and initial stretch $\lambda_{z 0}=1.6$.

Figure 18 shows a typical set of tube configurations as inflation proceeds, with Figures 19 and 20 showing the associated variation against time of pressure and outside radius at the crown of the bulge, respectively. Figure 21 displays the corresponding bulging profiles. Both Figures 19 and 20 show the fact that the pressure starts to rise as soon as it has reached a minimum, in contrast with the case of fixed $F$. In Figure 20 we have marked the theoretical 
predictions for the initiation pressure and the minimum pressure. The experimental initiation pressure 0.52 is less than the theoretical prediction 0.62 by about $16 \%$, whereas the experimental pressure minimum 0.32 is less than its theoretical counterpart 0.33 by $3 \%$.

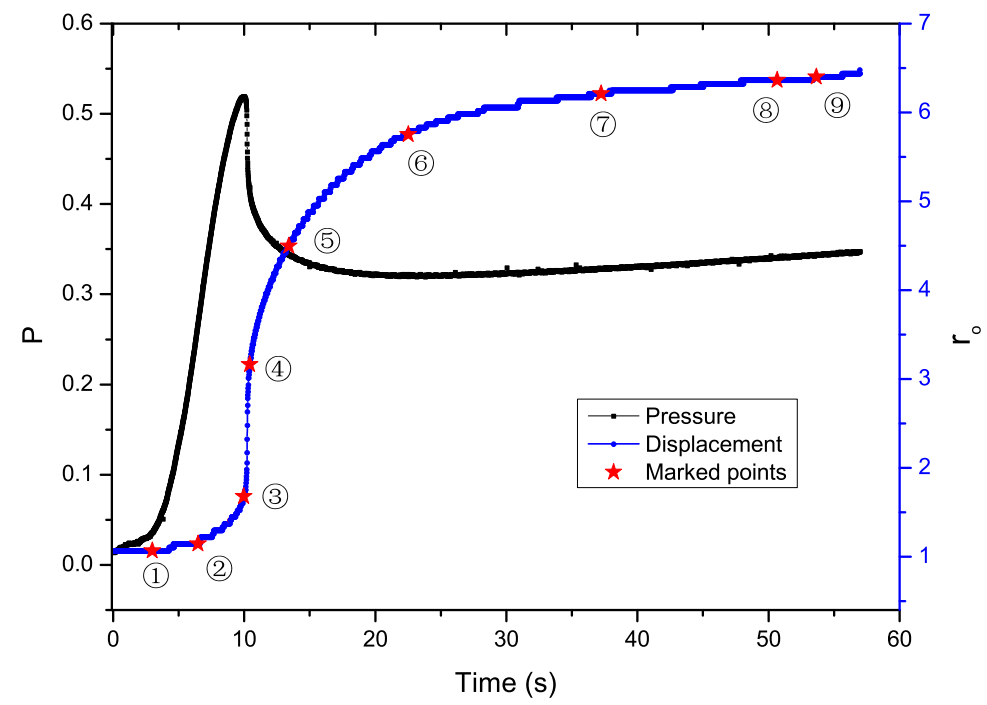

Figure 19: Evolution of pressure and bulge amplitude with time. The nine marked points correspond to the nine configurations in Figure 18.

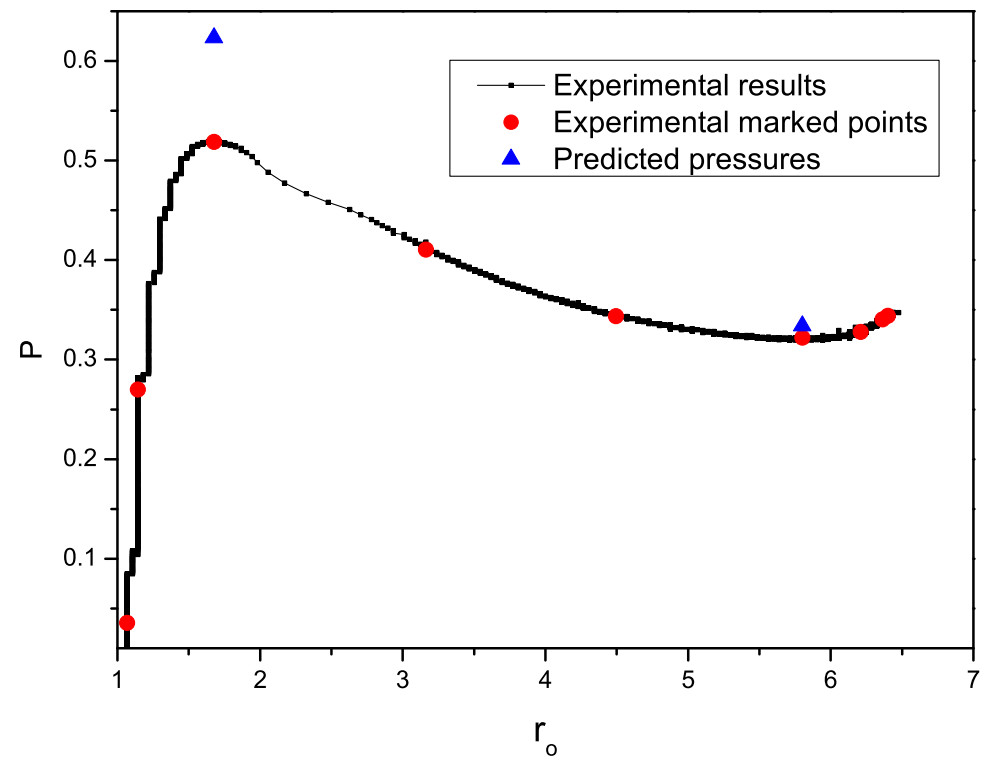

Figure 20: Dependence of pressure on bulge amplitude. The nine marked points correspond to the nine configurations in Figure 18. 


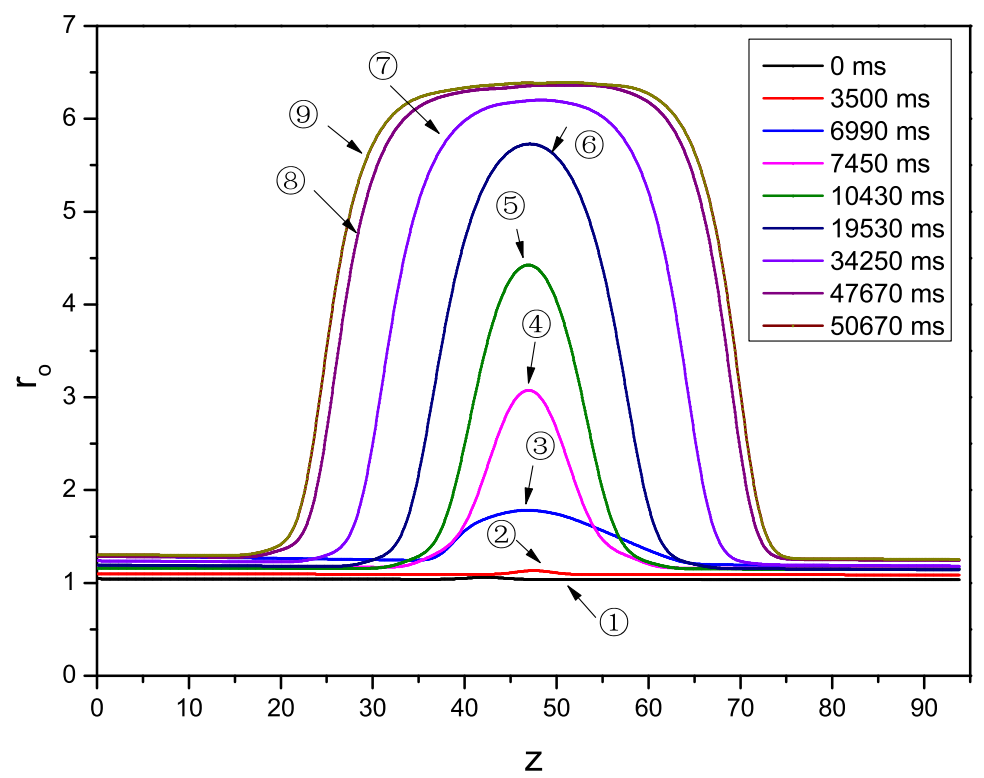

Figure 21: Bulge profiles corresponding to the nine configurations in Figure 18.

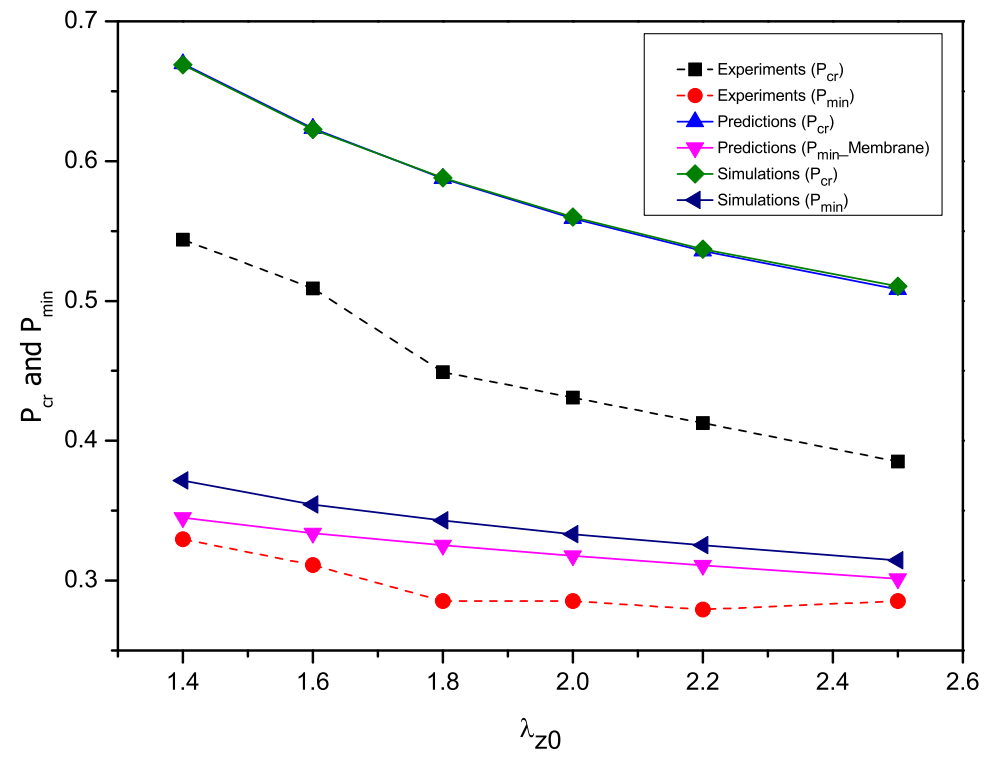

Figure 22: Dependence of the initiation and minimum pressures on $\lambda_{z 0}$ when $H=0.4, L=30$.

The next three figures show the dependence of the initiation and minimum pressures on the pre-stretch $\lambda_{z 0}$, the scaled wall thickness, and the scaled tube length. Figure 22 displays the effect of $\lambda_{z 0}$ for a tube of moderate wall thickness $(\varepsilon=0.4)$ that is chosen to have the same dimensions as the tube in Figure 15 for comparison. As in Figure 15, the experimental initiation pressure is consistently smaller than its theoretical counterpart by about $8 \%$ to $21 \%$. The theoretical predictions for the pressure minimum are slightly lower than the numerical simulation results. This is because the theoretical predictions are based on the membrane theory which tends to underestimate the pressure. It is also seen that the 
experimental minimum pressures are noticeably lower than the numerical simulation results. We are not able to suggest any convincing explanations since very little is known about the character of the pressure minimum. For instance, it is not known whether the pressure minimum is also sensitive to imperfections. Our experimental results seem to suggest that it is sensitive to imperfections, but to a less degree than the initiation pressure.

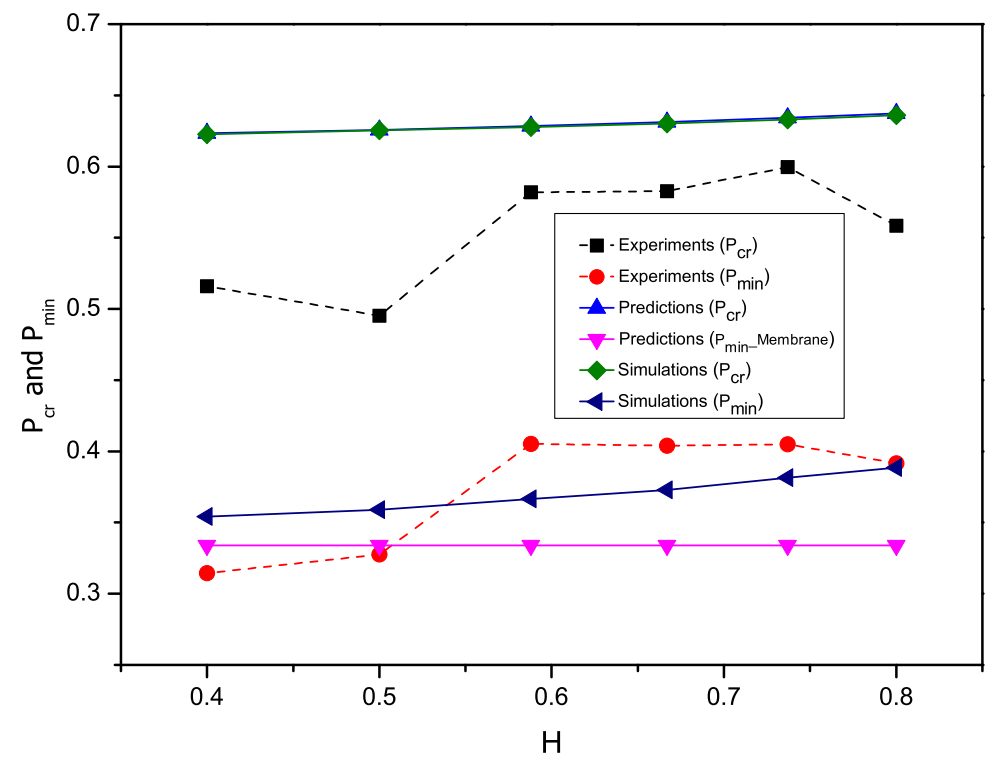

Figure 23: Dependence of initiation and minimum pressures on wall thickness when $L=30, \lambda_{z 0}=1.6$.

In Figure 23, we have shown the dependence of the two pressures on the scaled wall thickness. As the wall thickness increases, our numerical simulation results and the corresponding experimental results follow the same trend, but there are some minor fluctuations in our experimental results. Interestingly, the behaviour of various pressure values is very similar to the behaviour in Figure 16 for the other case. In particular, for the four larger values of $H$, the gap between the theoretical initiation pressure and its experimental counterpart is getting smaller, and the experimental minimum pressure lies slightly above the theoretical minimum pressure. The theoretical pressure minimum based on the membrane theory is independent of $H$, and as $H$ increases it gradually diverges from the numerical simulation results as expected.

Finally, we consider the dependence of the two pressures on the tube length. Figure 24 shows that when the tube length is larger than 20, both experimental and numerical simulation results become insensitive to the tube length. The initiation pressures given by theory and by experiments differ by about $8 \%$, the gap being slightly smaller than for lower values of $H$, as has already been observed in Figure 23. 


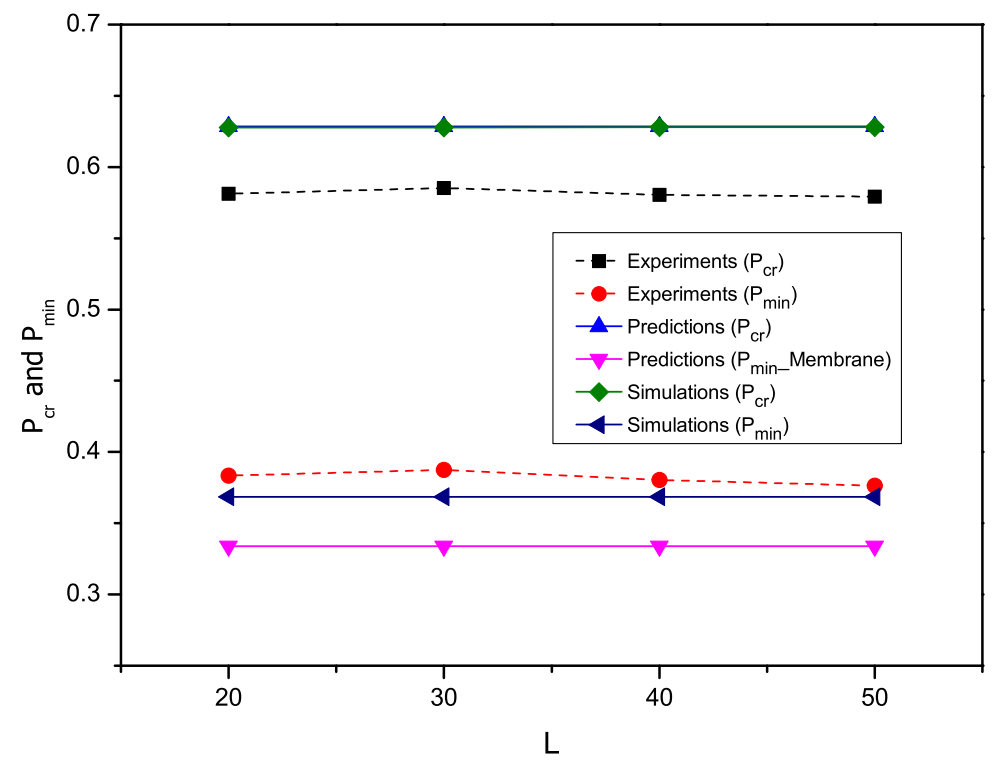

Figure 24: Dependence of the initiation and minimum pressures on the tube length when $H=0.59, \lambda_{z 0}=$ 1.6 .

\section{Conclusion}

Localized bulging of an inflated rubber tube is a classical problem in nonlinear elasticity, but it has been studied as a bifurcation problem only recently. In this paper, we have provided an updated summary of the newly emerged results, and have conducted a series of experiments to validate them. Complementing the existing experimental results, our current study has clarified the sensitivity of the initiation pressure to imperfections, and has provided a more precise description of how the bulge propagates in the axial direction, especially in the case when the axial length is fixed.

We focused on the case when the tube is sufficiently long so that the end conditions have a negligible effect on the initiation pressure. This is verified with the aid of numerical simulations using Abaqus. For tubes whose length is more than 40 times greater than the averaged radius (i.e. $L / R_{m}>20$ ), the initiation pressure obtained by Abaqus and the exact value based on an infinite tube differ from each other by less than $0.4 \%$. Once this is established, the only imperfections that are present are those arising from the manufacturing of the tubes. It is confirmed that the initiation pressure is indeed sensitive to imperfections: the values measured experimentally are consistently lower than the theoretical predictions by about $15 \%$. The largest gap occurs for tubes with the smallest wall thickness, which seems to suggest that localized bulging of thin-walled tubes is more sensitive to imperfections than thick-walled tubes. It is also confirmed that the scaled initiation pressure increases monotonically with respect to wall thickness, but the effect of bending stiffness is only marginal for thin and moderately thick tubes. These results are valid for both the case of fixed axial force and the case of fixed tube length. However, the pressure variation for the two different end conditions exhibit quite different behaviour. In the case of fixed axial 
force, the pressure first reaches a maximum at which the bulge initiates and then decreases monotonically to a constant value (the Maxwell pressure), whereas in the other case after reaching a maximum where a bulge initiates, the pressure decreases to a minimum and then rises monotonically. This is observed in our experiments and also predicted analytically. Our

experiments and numerical simulations confirm that the propagation pressure is insensitive to imperfections, but suggest that the pressure minimum in the case of fixed length might be sensitive to imperfections although to a less degree than the initiation pressure.

\section{Acknowledgements}

This work was supported by the National Natural Science Foundation of China (Grant Nos 11372212 and 11472186). We thank Mr. Lishuai Jin for his advice on the use of Abaqus.

\section{References}

1. An, L., Wang, F.F., Cheng, S.B., Lu, T.Q., Wang, T.J., 2015. Experimental investigation of the electro-mechanical phase transition in a dielectric elastomer tube. Smart Mater. Struct. 24, 035006.

2. Alexander, H., 1971. The tensile instability of an inflated cylindrical membrane as affected by an axial load. Int. J. Mech. Sci. 13, 87-95.

3. Alhayani, A.A., Giraldo, J.A., Rodríguez1, J., Merodio, J., 2013. Computational modelling of bulging of inflated cylindrical shells applicable to aneurysm formation and propagation in arterial wall tissue. Finite Elements in Analysis and Design 73, $20-29$.

4. Alhayani, A.A., Rodríguez, J., Merodio, J., 2014. Competition between radial expansion and axial propagation in bulging of inflated cylinders with application to aneurysms propagation in arterial wall tissue. Int. J. Eng. Sci. 85, 74-89.

5. Bucchi, A., Hearn, G.E., 2013a. Delay or removal of aneurysm formation in the Aanconda wave energy extraction device. Renewable Energy 55, 104-119.

6. Bucchi, A., Hearn, G.E., 2013b. Predictions of aneurysm formation in distensible tubes: Part A Theoretical background to alternative approaches. Int. J. Mech. Sci. $71,1-20$.

7. Chater, E., Hutchinson, J.W., 1984. On the propagation of bulges and buckles. ASME J. Appl. Mech. 51, 269-277.

8. Demirkoparan, H. and Merodio, J., 2017. Bulging bifurcation of inflated circular cylinders of doubly fiber-reinforced hyperelastic material under axial loading and swelling. Math. Mech. Solids. 22, 666-682.

9. Fu, Y.B., Il'ichev, A.T., 2015. Localized standing waves in a hyperelastic membrane tube and their stabilization by a mean flow. Maths Mech. Solids 20, 1198-1214.

10. Fu, Y.B., Pearce, S.P., Liu, K.K., 2008. Post-bifurcation analysis of a thin-walled hyperelastic tube under inflation. Int. J. Non-linear Mech. 43, 697-706. 
11. Fu, Y.B., Xie, Y.X., 2010. Stability of localized bulging in inflated membrane tubes under volume control. Int. J. Eng. Sci. 48, 1242-1252.

12. Fu, Y.B., Xie, Y.X., 2012. Effects of imperfections on localized bulging in inflated membrane tubes. Phil. Trans. R. Soc. A370, 1896-1911.

13. Fu, Y.B., Rogerson, G.A., Zhang, Y.T., 2012. Initiation of aneurysms as a mechanical bifurcation phenomenon. Int. J. Non-linear Mech. 47, 179-184.

14. Fu, Y.B., Xie, Y.X., Dorfmann, L., 2018. Localized necking of a dielectric membrane. Extreme Mechanics Letters 21, 44-48.

15. Fu, Y.B., Liu, J.L., Francisco, G.S., 2016. Localized bulging in an inflated cylindrical tube of arbitrary thickness - the effect of bending stiffness. J. Mech. Phys. Solids 90, 45-60.

16. Gent, A.N., 2005. Elastic instabilities in rubber. Int J Non-Linear Mech. 40, 165-75.

17. Guo, Z.M., Gattas, J., Wang, S.B., Li, L.A., and Albermani, F., 2016. Experimental and numerical investigation of bulging behaviour of hyperelastic textured tubes. Int. J. Mech. Sci. 115-116, 665-675.

18. Goncalves, P.B., Pamplona, D.C., Lopes, S.R.X., 2008. Finite deformations of an initially stressed cylindrical shell under internal pressure. Int. J. Mech. Sci. 50, 92-103.

19. Haughton, D.M., Ogden, R.W., 1979. Bifurcation of inflated circular cylinders of elastic material under axial loading. II. Exact theory for thick-walled tubes. J. Mech. Phys. Solids 27, 489-512.

20. Horny, L., Netusil, M, Horak, Z., 2015. Limit point instability in pressurization of anisotropic finitely extensible hyperelastic thin-walled tube. Int. J. Non-linear Mech. $77,107-114$.

21. Kanner, L.M., Horgan, C.O., 2007. Elastic instabilities for strain-stiffening rubber-like spherical and cylindrical thin shells under inflation. Int. J. Non-linear Mech. 42, 204-215.

22. Kyriakides, S., Chang, Y.-C., 1990. On the inflation of a long elastic tube in the presence of axial load. Int. J. Solids Struct. 26, 975-991.

23. Kyriakides, S., Chang, Y.-C., 1991. The initiation and propagation of a localized instability in an inflated elastic tube, Int. J. Solid Struct. 27, 1085-1111.

24. Lestringant, C., Audoly, B., 2018. A diffuse interface model for the analysis of propagating bulges in cylindrical balloons. Proc. Roy. Soc. A 474, 20180333.

25. Lu, T.Q., An, L., Li, J.G., Yuan, C. and Wang, T.J., 2015. Electro-mechanical coupling bifurcation and bulging propagation in a cylindrical dielectric elastomer tube. J. Mech. Phy. Solids. 85 (2015), 160-175.

26. Mallock, A., 1891. Note on the instability of India-rubber tubes and balloons when distended by fluid pressure. Proc. Roy. Soc. A 49, 458-463.

27. Mangan, R., Destrade, M., 2015 Gent models for the inflation of spherical balloons. Int. J. Non-linear Mech. 68, 52-58. 
28. Mao, G.Y., Li T.F., Zou, Z.N., Qu, S.X., Shi, M.X., 2014. Prestretch effect on snapthrough instability of short-length tubular elastomeric balloons under inflation. Int. J. Solids Struct. 51, 2109-2115.

29. Ogden, R.W., Saccomandi, G., Sgura, I., 2004. Fitting hyperelastic models to experimental data. Comput Mech. 34, 484-502.

30. Pamplona, D.C., Goncalves, P.B., Lopes, S.R.X., 2006. Finite deformations of cylindrical membrane under internal pressure. Int. J. Mech. Sci. 48, 683-696.

31. Pearce, S.P., Fu, Y.B., 2010. Characterisation and stability of localised bulging/necking in inflated membrane tubes. IMA J. Appl. Math. 75, 581-602.

32. Pearce, S.P., 2012. Effect of strain-energy function and axial prestretch on the bulges, necks and kinks forming in elastic membrane tubes. Math. Mech. Solids, 17 , 860-875.

33. Pipkin, A.C.,1968. Integration of an equation in membrane theory. ZAMP 19, 818819.

34. Pucci, E, Saccomandi, G., 2002. A note on the gent model for rubber-like materials. Rubber Chem Technol, 75, 839-52.

35. Ren, J.S., 2007. Inflation of an artery leading to aneurysm formation and rupture. Mol. Cell Biomech. 4, 55-66.

36. Rodrguez-Martinez, J.A., Fernndez-Sez, J., Zaera, R., 2015. The role of constitutive relation in the stability of hyper-elasticspherical membranes subjected to dynamic inflation. Int. J. Eng. Sci. 93, 31-45.

37. Shi, J., Moita, G.F., 1996. The post-critical analysis of axisymmetric hyper-elastic membranes by the finite element method. Comput. Methods Appl. Mech. Engrg. $135,265-281$.

38. Takla, M., 2018. Bifurcation of elastic-plastic thick-walled cylindrical structures. Int. J. Mech. Sci. 141, 303-315.

39. Treloar, L.R.G., 1944a. Stree-strain data for volcanized rubber under various types of deformation. Trans. Faraday Soc. 40, 59-70.

40. Treloar, L.R.G., 1944b. Strains in an inflated rubber sheet and the mechanism of bursting. Rubber Chem. Technol. 19, 201-212.

41. Varatharajan, N., DasGupta, A., 2017. Study of bifurcation in a pressurized hyperelastic membrane tube enclosed by a soft substrate. Int. J. Non-linear Mech. 95, 233-241.

42. Wang, F.F., Chao, Y., Lu, T.Q., Wang, T.J., 2017. Anomalous bulging behaviors of a dielectric elastomer balloon under internal pressure and electric actuation. J. Mech. Phys. Solids 102, 1-16.

43. Wang, J., Fu, Y.B., 2018. Effect of double-fibre reinforcement on localized bulging of an inflated cylindrical tube of arbitrary thickness. J. Eng. Math. 109, 21-30.

44. Wang, T., Xu, F., Huo, Y., Potier-Ferry, M., 2018. Snap-through instabilities of pressurized balloons: Pear-shaped bifurcation and localized bulging. Int. J. Nonlinear Mech. 98, 137-144. 
45. Wineman, A.S., 2015. Determining the time of bulge formation in an elastomeric tube as it inflates, elongates and alters chemorheologically. Math. Mech. Solids 20, 9-24.

46. Wineman, A.S., 2017. Bulge initiation in tubes of time-dependent materials. Math. Mech. Solids 22, 636-648.

47. Wolfram Research, Inc., 2015. Mathematica, Version 10.2, Champaign, Illinois.

48. Yin, W.-L., 1977. Non-uniform inflation of a cylindrical elastic membrane and direct determination of the strain energy function. J. Elast. 7, 265-282.

49. Zhou, L., Wang, S.B., Li, L.A., Fu, Y.B., 2018. An evaluation of the Gent and GentGent material models using inflation of a plane membrane. Int. J. Mech. Sci. 146-147, $39-48$. 